\title{
ANÁLISIS DE MiCROVESTIGIOS EN PIPAS PROCEDENTES DE OCUPACIONES PREHISPÁNICAS DE LAS SELVAS MERIDIONALES DEL noroeste Argentino. El CASO de YÁnimas 1
}

\author{
Valeria B. Martín Silva', Gabriel E. Miguez y M. Alejandra Korstanje $e^{1,2}$
}

\begin{abstract}
Resumen
En este trabajo se exponen los resultados obtenidos a partir del análisis efectuado sobre residuos adheridos en fragmentos de pipas de cerámica recuperados en las elevaciones monticulares I (EM1) y 3 $\left(\mathrm{EM}_{3}\right)$ del Sitio Yánimas 1. Para llevar a cabo este estudio se realizó la preparación de muestras a partir del residuo recuperado en fragmentos de pipas (tubos de aspiración y hornillo, en el caso de la muestra F del sector EM 3) para su observación en microscopio petrográfico y posterior comparación con el material botánico actual de referencia. De este modo se pudieron efectuar inferencias taxonómicas de los microvestigios observados. Los análisis llevados a cabo permitieron constatar la presencia de granos de almidones símil Nicotiana spp., y granos de almidón y calcifitolitos afines a Anadenanthera colubrina var. cebil (Griseb.) Altschul. A través de este trabajo, se pudo aportar nuevos datos sobre la utilización de pipas y el consumo de plantas enteógenas en el piedemonte tucumano a fines del primer milenio DC.
\end{abstract}

Palabras claves: microrrestos - piedemonte tucumano pipas prehispánicas - plantas enteógenas.

\begin{abstract}
In this paper we expose the results obtained from the residues analysis in ceramic pipe's fragments recovered from mound elevation 1 (EMI) and ${ }_{3}\left(\mathrm{EM}_{3}\right)$ at the site, Yánimas 1 . To carry out this study we performed the preparation of samples from the residues taken from the pipe fragments (suction pipes and stove pipes in the case of Sample F for the sector $\mathrm{EM}_{3}$ ) in order to observe them at petrographic microscope. We compared the results with the current botanical reference material.

In this way it was possible to make some taxonomic inferences of the observed microremains. This methodology threw positive results for residues showing the presence of starch grains simile to Nicotiana spp. and starch grains and calcium phytoliths possibly ascribed to Anadenanthera

colubrina var. cebil (Griseb.) Altschul. Through this analysis we could discuss new data regarding the use of pipes and consumption of entheogenic plants in Tucumán's piedmont, at late first millennium AD.
\end{abstract}

Key words: microremains - southern yungas - smoking pipes entheogenic plants.

Recibido: Marzo 2015. Aceptado: Abril 2016

\section{* Introducción}

En tiempos precoloniales, las pipas tuvieron amplia distribución en el Noroeste Argentino (NOA). Se las ha registrado en numerosos y variados contextos arqueológicos, pero con mayor frecuencia en emplazamientos ubicados en el área Valliserrana, en la Puna y en las tierras bajas de Jujuy y Salta. Sobre varios de estos ejemplares se han efectuado estudios formales, contextuales $y$, más recientemente, análisis de sus residuos internos, que en conjunto han contribuido de manera significativa al conocimiento de las tecnofacturas tradicionalmente utilizadas por las comunidades prehispánicas de dichas áreas, como así también sobre las plantas consumidas en las prácticas fumatorias (Bregante 1926; Boman 1932; Serrano 1934; Ryden 1936; Krapovickas 1955; Heredia 1970; Dougherty 1972; González 1977; Fernández Distel 1980; Pérez Gollán y Gordillo 1993; Fernández Distel et al. 1999; Pochettino y Capparelli 2004; Capparelli et al. 2006; Lema et al. 2015; entre otros).

Sin embargo, poco es lo que se sabe acerca de estos artefactos y sus contextos arqueológicos en los ambientes selváticos meridionales del NOA, como tampoco sobre las prácticas involucradas en su utilización. Por ejemplo, ¿cuáles eran las características formales de estos artefactos? o ¿qué plantas eran empleadas en el hábito de fumar $y$ con qué fin? Y en cuanto a las plantas utilizadas, ifueron obtenidas localmente o a través de intercambios en el marco de redes de tráfico? Por ello, el principal objetivo de este trabajo es lograr un acercamiento a la identificación de las plantas consumidas a través de prácticas fu-

1 Instituto de Arqueología y Museo (UNT); San Martín 1545, 4000 San Miguel de Tucumán, Tucumán, Argentina. Email: belenita8@gmail.com; gabrielmiguez7@hotmail.com.

2 Instituto Superior de Estudios Sociales (CONICET), San Lorenzo 429, CP 4000, San Miguel de Tucumán, Tucumán, Argentina. Email: ale_korstanje@hotmail.com. 
matorias llevadas a cabo en ocupaciones prehispánicas del piedemonte meridional de Tucumán. Para ello, realizamos el análisis microscópico de los residuos internos de fragmentos de pipas procedentes del sitio Yánimas 1 (ca. 750-1200 DC) (Figura 1). Luego, y a partir de los resultados obtenidos, indagamos sobre las posibles prácticas involucradas en el aprovisionamiento, procesamiento y consumo de las plantas identificadas en las pipas estudiadas, tomando en consideración la información etnográfica y arqueológica pertinente al tema, existente tanto para el NOA como también para áreas aledañas como la Región Chaqueña.

Por último, se exploran las relaciones entre estos artefactos y los contextos de procedencia, con el motivo de discutir el papel que habrían tenido las prácticas fumatorias en diferentes lugares de esta ocupación prehispánica. ¿Cuáles eran los espacios donde se desenvolvieron tales prácticas?, y icuáles fueron las implicancias sociales vinculadas con la obtención y consumo de las plantas identificadas? Resulta relevante indagar sobre estas cuestiones ya que no solo aportarían datos acerca de las actividades involucradas en los hábitos fumatorios, sino que también podrían aportar información que contribuya a comprender mejor los espacios de este sitio y las prácticas desarrolladas en ellos hacia fines del primer milenio DC.

\section{El consumo de plantas enteógenas en los tiempos prehispánicos del NOA}

A diferencia de los que ocurre en los Andes Centrales, donde la presencia de pipas es muy escasa, en el NOA han sido reportadas en diversos contextos arqueológicos. Como resultado de ello, en la actualidad existe una amplia colección de estos artefactos, de una importante variedad de formas y tamaños, los cuales han sido confeccionados sobre diversas materias primas (hueso, piedra y cerámica), universo dentro del cual se pueden distinguir ciertas tradiciones estilísticas (pipas tubulares, monitor, acodadas, etc.). Pero más allá de los materiales y estilos vinculados a la producción de estos artefactos, nos interesa profundizar particularmente en las posibles plantas

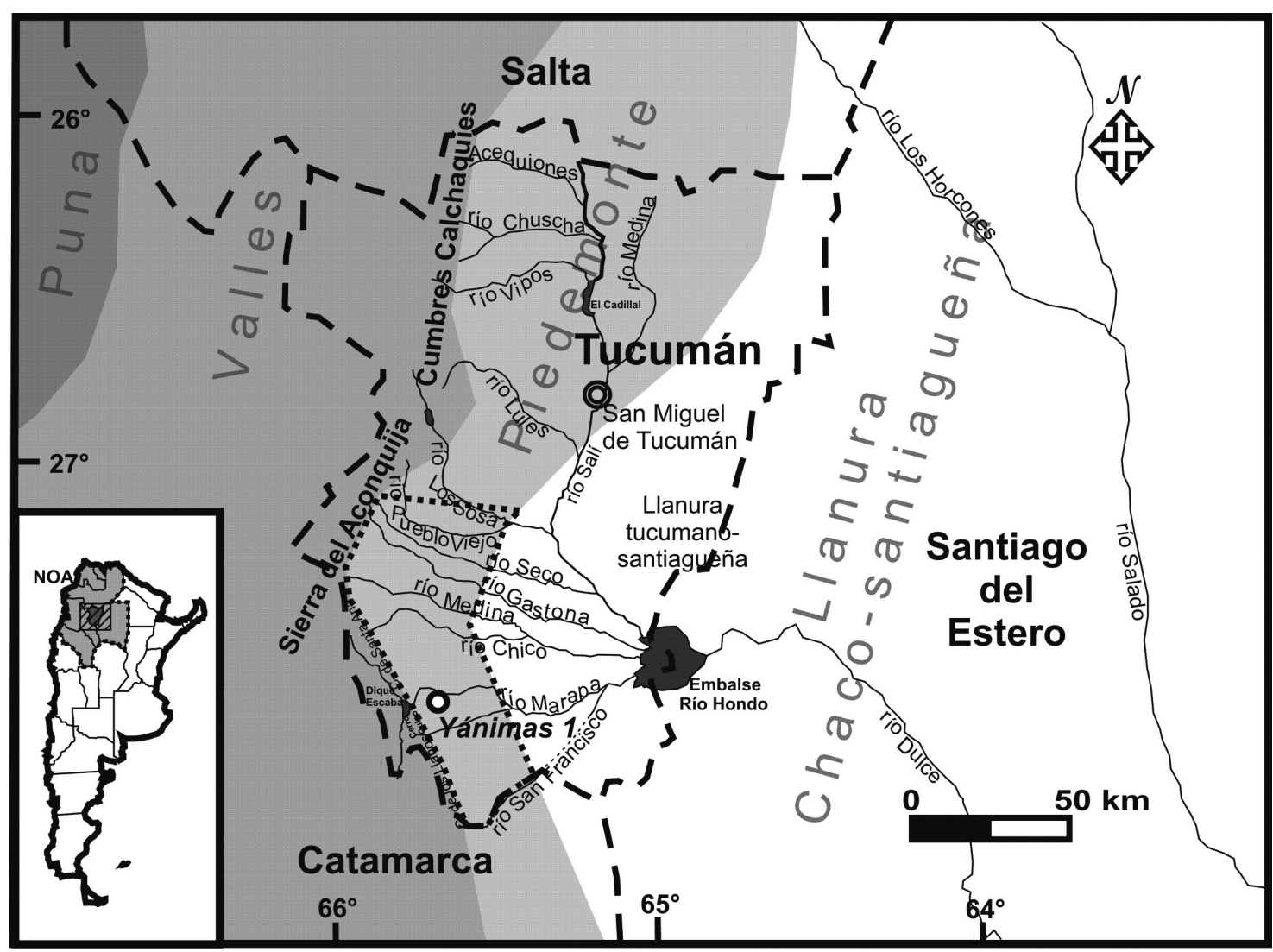

Figura 1. Ubicación del sitio Yánimas l y delimitación del área de estudio (en línea de puntos). 
consumidas y las metodologías aplicadas para su identificación, así como también los hábitos relacionados con estas prácticas.

En la historiografía referida al tema, se pueden observar interesantes cambios en los enfoques y métodos utilizados. Hasta fines del siglo pasado fueron relativamente pocos los autores que han puesto su atención en estos artefactos. Ejemplos de ello han sido los trabajos de Nordenskiöld 1993 [1903]; Bregante 1926; Boman 1932; Serrano 1934; Márquez Miranda 1946; González 1963, 1961-1964, 1977, 1998; Dougherty 1972, 1977; Fernández Distel 1980; Pérez Gollán y Gordillo 1993, 1994; y Constantino 1998. Estos trabajos se enfocaron principalmente en la descripción de las características formales de las pipas y sus contextos de hallazgo (para el caso de los ejemplares hallados en intervenciones arqueológicas, ya que buena parte de los especímenes estudiados proceden de colecciones arqueológicas, con escasas referencias contextuales). Con excepción de Bregante (1926), quien sostiene que pudieron ser utilizadas como incensarios, en general se acepta que estos objetos fueron empleados para fumar sustancias psicoactivas ${ }^{3}$. Asimismo, la mayoría de los autores citados recurrieron a las fuentes etnohistóricas y/o etnográficas, para sugerir que las especies consumidas en estos artefactos serían, principalmente, plantas asociadas a tres géneros: el cebil [Anadenanthera colubrina var. cebil (Griseb.) Altschul], el coro (Nicotina sp. o Tricholine spp.), y el tabaco (Nicotiana spp.).

Fue Fernández Distel (1980) quién aportó los primeros datos concretos obtenidos a partir de técnicas de laboratorio sobre el consumo de sustancias en pipas prehispánicas. Mediante el análisis químico (por cromatografía gaseosa) de los residuos internos contenidos en un conjunto de este tipo de artefactos recuperados en los sitios precerámicos Inca Cueva 7 y Huachichocana III (ambos situados en la Puna de Jujuy y ubicados cronológicamente entre ca. 4000-3000 años AP), la autora señala que en el primer caso (dos pipas fabricadas a partir de hueso de Felis concolor) se pudo determinar la presencia de un alcaloide (bufotenina) que es propio del cebil [Anadenanthera colubrina var. cebil (Griseb.) Altschul], mientras que en el segundo caso (cuatro pipas de piedra) se detectaron varios alcaloides (ninguno compatible con la bufotenina o nicotina), posiblemente correspondientes a varias especies vegetales ${ }^{4}$ (Fernández Distel 1980). Otro aporte fundante fue el de Pochettino et al. (1999) que, si bien no fue realizado sobre pipas, constituyó una importante contribución a esta problemática desde lo metodológico. En este estudio, a partir de la observación al microscopio del material pulverizado extraído del interior de un cubilete de hueso proveniente del sitio alero La Matanza 1 (Jujuy), se identificó la presencia de microrrestos que, por sus características formales, pertenecerían al cebil. En relación al contexto de hallazgo, el mencionado cubilete fue recuperado en asociación a dos cuerpos momificados, formando parte del ajuar característico del complejo de rape o complejo alucinógeno ${ }^{5}$. Ambos trabajos fueron relevantes porque abrieron nuevas vías de análisis para una identificación más directa del universo de plantas y sustancias consumidas en las pipas prehispánicas del NOA (o en otros objetos), las cuales tuvieron mayor desarrollo en momentos posteriores.

Más recientemente se observa un renovado interés sobre estos artefactos (Planella et al. 2000; Falabella et al. 2001; Pochettino y Capparelli 2004; Rosso y Spano 2005-2006; Capparelli et al. 2006; Muñoz y Peña 2009; Andreoni 2010; Bugliani et al. 2010; Andreoni et al. 2012; Quiroz et al. 2012; Planella et al. 2012; Lema et al. 2015; entre otros), fundamentalmente orientado a la identificación de las plantas enteógenas utilizadas a partir del estudio de los residuos internos adheridos a las pipas. Sobre estos residuos se han aplicado diferentes metodologías arqueobotánicas, como la determina-

3 A propósito, es preciso señalar que recientemente Velandia Jagua (2014) ha reavivado el debate sobre la problemática de la función de las pipas del NOA. Este autor ha propuesto que estos objetos, o al menos un conjunto de ellos (las pipas de piedra o cerámica acodadas, con orificio en el vértice de unión de los brazos del tubo-boquilla y la hornilla), no habrían sido artefactos para fumar, sino "clísteres para la aplicación de enemas alucinógenos" (Velandia Jagua 2014: 69).

4 Sin embargo, en un reciente trabajo (Lema et al. 2015) se estudiaron dos de estas pipas (códigos 2037 y 2038) mediante la extracción de residuos internos. La recuperación de microrrestos y el análisis en microscopio, permitió la identificación de microrrestos de cebil (Anadenanthera colubrina var. cebil) y tabaco (Nicotiana longiflora y Trichocline reptans).

5 La cronología de este hallazgo data de 1384 DC, fechado obtenido a partir del análisis de fosfatasa alcalina de costillas de unos de los cuerpos encontrados en asociación al complejo fumatorio (Fernández Distel 1997). 
ción química de sustancias por cromatografía gaseosa y espectrometría de masa, la identificación de distintos tipos de microrrestos (extraídos mediante la aplicación de diferentes protocolos) a partir de la observación de muestras en microscopio, o la realización de análisis para la detección de ácidos grasos. Al respecto, cabe señalar que si bien en algunos casos fue posible identificar el uso de enteógenos afines al género Nicotiana u otros como Anadenanthera colubrina var. cebil (Griseb.) Altschul (Bugliani et al. 2010; Rosso y Spano 2005-2006; Planella et al. 2012; Quiroz et al. 2012; Lema et al. 2015), en otros no pudo determinarse su presencia (Andreoni et al. 2012). Sin embargo, hay que destacar que estos estudios ampliaron el espectro de especies que pudieron haber sido empleadas en el hábito de fumar y sus posibles finalidades (suavizantes, sucedáneas, aromáticas, entre otras, además de las narcotizantes), lo que ha significado un considerable avance en el conocimiento acerca de la variabilidad y complejidad de las actividades involucradas en estas costumbres (p.e. Capparelli et al. 2006). Asimismo, la consulta de fuentes etnográficas también ha sido fundamental para comprender las posibles maneras en que las diversas plantas identificadas pudieron haber sido utilizadas (por ejemplo, el empleo de mezclas o el uso de filtros) o, incluso, para explorar las razones por las cuales ciertos residuos -no relacionados con el propio acto de fumar- fueron hallados en el interior de las pipas (Andreoni et al. 2012). Incluso recientemente se ha planteado que el análisis exhaustivo de estas fuentes podría enriquecer aún más este universo, permitiéndonos ahondar en los procesos de selección y utilización de los diferentes tipos de plantas, ya sea con o sin compuestos psicoactivos (Andreoni 2010).

Por otra parte, también se ha avanzado en la comprensión de los espacios y las esferas sociales en los que estos objetos fueron utilizados. Tradicionalmente se ha considerado a las pipas como elementos con un importante carácter simbólico y su uso ha sido con frecuencia asociado a prácticas rituales y lugares sagrados (González 1977, 1998; Pérez Gollán y Gordillo 1993, 1994; entre otros). Recientemente se han cuestionado estas interpretaciones que simplifican el uso y significado de estos artefactos y de las sustancias consumidas en ellos, planteándose que el hábito de fumar no estuvo siempre asociado a los espacios rituales, ya que se ha registrado el uso de pipas en contextos domésticos (Bugliani et al. 2010; Palamarczuk et al. 2007; Rosso y Spano 2005-2006). Tampoco el uso de estos objetos implicaría necesariamente un acto sagrado, dado que incluso se ha sugerido el consumo de especies vegetales sin propiedades psicotrópicas (Capparelli et al. 2006). En todo caso, como señala Babot (2009), dependiendo del grupo humano que las ha empleado, varias plantas como así también los artefactos en los que fueron procesadas o consumidas -en este caso, las pipas- pudieron haber adquirido propiedades utilitarias y/o sagradas en distintos momentos y espacios de uso, para lo cual hay que tener en cuenta los contextos y actividades en los que se encontraban involucrados a lo largo de sus trayectorias de vida. Por ejemplo, en comunidades originarias de la Región Chaqueña argentina (mocovíes), Scarpa y Rosso (2011) han registrado el consumo del coro -que según estos autores sería una especie silvestre de tabaco, Nicotiana paa (Mart. Crov.)- en pipas tubulares de arcilla tanto en prácticas rituales y celebraciones multitudinarias como en forma personal en la intimidad de los ámbitos domésticos. Además estos autores señalan que esta planta ha sido empleada no solo como estimulante en actividades rituales, sino también como fumatorio, mascatorio, insectífugo y con fines medicinales.

\section{EL CASO DE ESTUDIO: EL SITIO YÁNIMAS I}

El sitio Yánimas 1, que fuera dado a conocer por Manasse (1997: 145) bajo la denominación Finca Juárez, forma parte del rico patrimonio arqueológico que posee la cuenca del río Marapa y zonas aledañas (véase Manasse 1997; Pantorrilla y Núñez Regueiro 2006; Rendace et al. 2006; Miguez et al. 2013; Miguez y Caria 2015). Está ubicado a unos $9 \mathrm{~km}$ al SW de la localidad de Alberdi, en el departamento de La Cocha, provincia de Tucumán (Figura 1). Emplazado en un ambiente de selva pedemontana de Yungas, sobre la margen meridional del río Marapa, posee una extensión aproximada de 45 hectáreas y, entre sus características más notables, se destacan un conjunto de prolongadas elevaciones monticulares y la abundancia y variabilidad de restos culturales asociados (Manasse 1997; Miguez 2010). Por ello, Yánimas 1 reviste gran relevancia para un área que ha sido poco explorada desde el punto de vista arqueológico. Es precisamente en este sitio donde se registran los primeros restos de pipas en contexto del piedemonte meridional tucumano.

Los primeros ejemplares fueron obtenidos en el marco 
de un rescate arqueológico efectuado por un equipo de profesionales del Instituto de Arqueología y Museo de la Universidad Nacional de Tucumán (Martel et al. 2007). En dicha intervención se recuperaron numerosos restos culturales que fueron afectados por la acción de una pala mecánica en uno de los montículos, entre los que destacan seis fragmentos de pipas. Posteriormente, en el contexto de recientes investigaciones arqueológicas desarrolladas en el piedemonte meridional de la provincia de Tucumán, se han llevado a cabo nuevas intervenciones en este sitio (prospecciones y excavaciones) en las que se han recuperado nuevos restos de pipas tanto en superficie como en estratigrafía. Pero además de ello, se han registrado interesantes contextos con abundantes y diversos materiales -incluso varios de naturaleza orgánica en buen estado de preservación- (Miguez 2010; Miguez et al. 2013; Miguez y Caria 2015; entre otros), que serán descritos brevemente a continuación, poniendo énfasis en los lugares donde proceden las pipas analizadas.
En primer lugar debemos señalar que, debido a su gran extensión, Yánimas I fue dividido en varios sectores. Los montículos del sitio se concentran en el Sector Central (en adelante, SC). Allí se relevaron 3 elevaciones monticulares alargadas (EM2, $\mathrm{EM}_{3}$ y EM4, que promedian 130 $\mathrm{m}$ de longitud y 1,5 m de altura), las cuales se encuentran unidas de tal manera en el terreno que forman una $\mathrm{U}$ abierta al suroeste y delimitan un espacio plano central de poco más de 1 ha. Estrechamente vinculado a la mitad occidental de la EM2, se eleva un cuarto montículo que posee menor extensión (EMl), pero se destaca por su altura $(3 \mathrm{~m})$ y su forma de cono truncado de planta oval (Figura 2). Por fuera del SC, se observa una extensa superficie ondulada con una distribución regular de restos arqueológicos, que en gran parte han sido removidos de su posición original por los desmontes y las tareas de arado. Las intervenciones arqueológicas efectuadas en el sitio (inspecciones superficiales, sondeos, rescates y excavaciones sistemáticas) han permitido definir la pre-

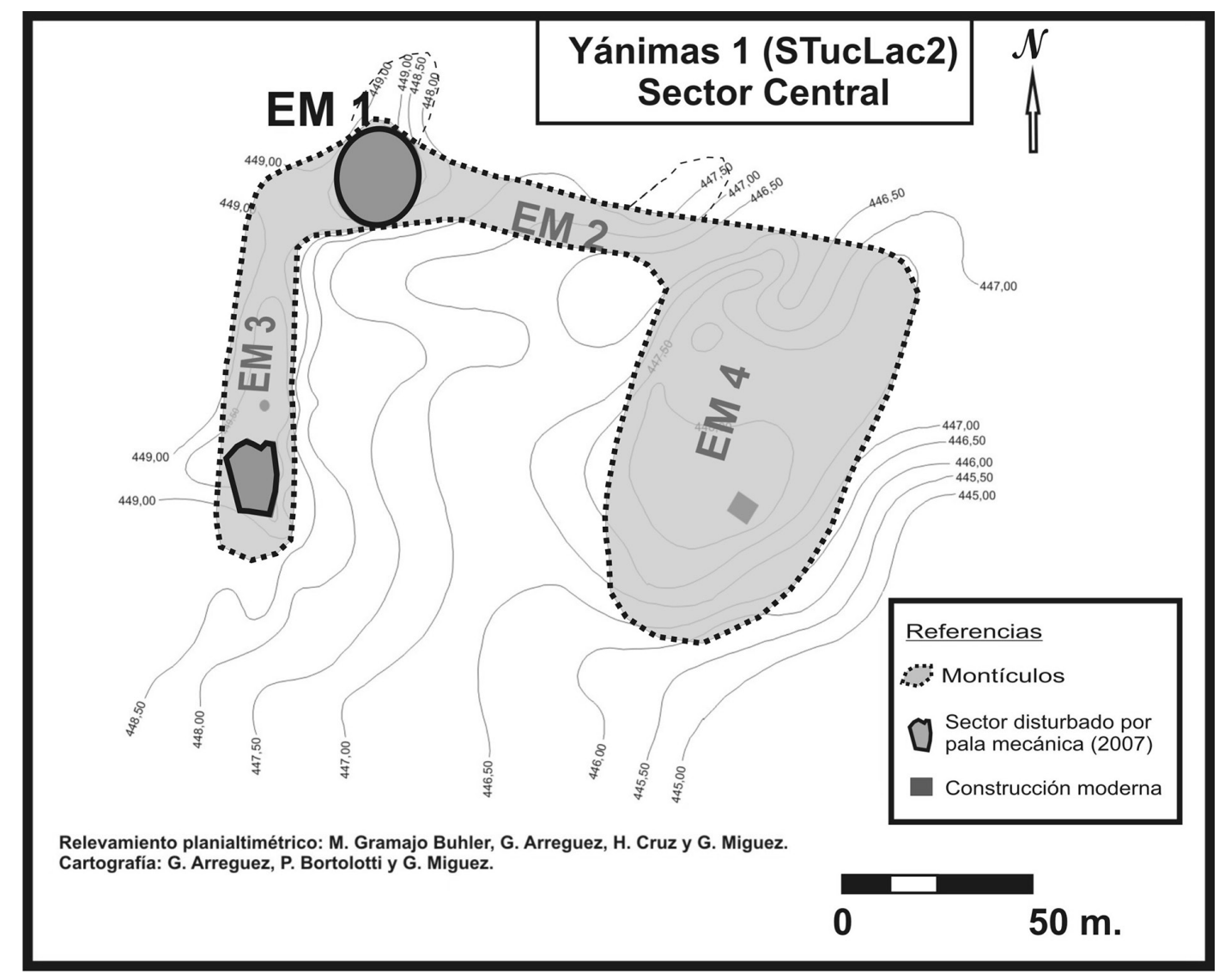

Figura 2. Plano del Sector Central del sitio y sus elevaciones monticulares. (Tomado de Miguez et al. 2012). 
sencia de, al menos, una ocupación prehispánica que cronológicamente se ubica hacia fines del primer milenio de la era cristiana (ca. 750-1200 DC) según los fechados obtenidos hasta el momento ${ }^{6}$. Sin embargo, es probable que la zona que abarca el sitio y sus alrededores haya sido ocupada en más de una oportunidad a lo largo del primer milenio DC (Miguez 2014).

El montículo EM i fue parcialmente excavado $(130 \mathrm{~cm}$ de profundidad máxima), obteniéndose un registro que presenta algunas características particulares en relación a otros espacios intervenidos, tales como la variabilidad de restos arqueológicos, la forma en que se distribuyen y la matriz sedimentaria. Los materiales más abundantes son fragmentos de vasijas, vestigios faunísticos (algunos con signos de haber sido expuestos al fuego) y macrorrestos vegetales carbonizados, generalmente muy bien preservados. Con menor frecuencia se recuperaron restos líticos tallados y unos pocos fragmentos de artefactos de roca pulida. Como hallazgos especiales podemos mencionar cuatro tubos de pipas, dos dientes humanos, tres cuentas de collar, dos laminillas de oro, un silbato óseo y tres piedras bezoares.

Aunque en general estos vestigios se presentan de forma dispersa, se han registrado algunas concentraciones interesantes en varios niveles excavados. Una concentración que puede ser relevante a este trabajo fue observada en el nivel $5(40-50 \mathrm{~cm})$ de las unidades 4 y 6 , donde se reconoció una asociación que se compone de varios tiestos, dos fragmentos de tubos de pipas, caparazones de moluscos terrestres, cáscaras de huevo de Rhea sp., algunas lascas, restos de camélidos, aves, carnívoros y otros animales, además de un bezoar y un aerófono elaborado con el fémur de un ave rapaz (Miguez et al. 2013; Nasif y Miguez 2014). En el mismo nivel pero en la unidad 8, se halló un tercer tubo de pipa asociada a otros vestigios (Figura 3). Estos materiales y asociaciones se encontraban depositados en una matriz de sedimentos sueltos, secos, ligeramente alcalinos y con granulometría francoarcillo-arenoso. Otra particularidad de este montículo es la presencia, en la mayoría de los niveles excavados,

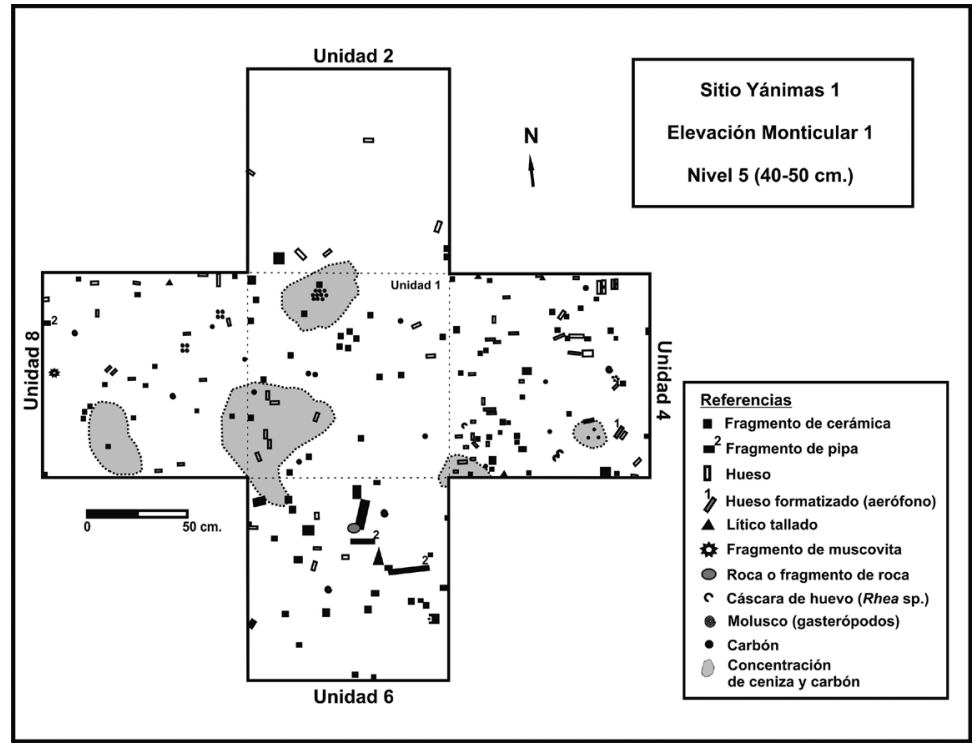

Figura 3. Gráfico de planta del Nivel 5 de todas las unidades excavadas en EM i (Tomado de Miguez et al. 2013).

6 Cabe aclarar aquí que en varios trabajos anteriores (Miguez et al. 2013; Miguez 2014; Miguez y Caria 2015; entre otros) se propuso una cronología de 900-1200 DC, en base a solo dos fechados publicados en dichos artículos. Sin embargo, recientemente se han obtenido nuevas dataciones para el sitio, que proceden específicamente del montículo EMı. Se trata de dos fechados cuyos resultados son muy similares, los cuales extenderían el límite inferior de la anterior cronología asignada del sitio hasta el 750 DC, o sea que ahora el sitio se ubicaría entre ca. 750-1200 DC. A los intereses de este trabajo, resulta relevante que uno de los fechados procede de una muestra ósea animal del nivel 5 (40-50 cm) de la Unidad 1, que se recuperó en posición cercana a los fragmentos de pipas A y B (y sus restos asociados) analizados en este trabajo. Dicho fechado dio como resultado 1240 +/- 25 años AP (UGAMS25525, hueso, $\delta_{13}$ Ccol.:-18,23 \%o). 
de abundantes carbones y manchas blanquecinas de aspecto ceniciento. Durante la excavación se han tomado muestras de sedimentos sobre las que fueron llevados a cabo análisis químicos (P y MO). Los resultados manifiestan una variación irregular (aumentos y descensos) de estos componentes a lo largo de la secuencia arqueoestratigráfica. Un fenómeno similar ocurre con los materiales culturales, ya que varios materiales (cerámicos, líticos y otros de origen orgánico) manifiestan vaivenes en sus frecuencias en relación a la profundidad. Las propiedades del registro arqueológico de EM 1 sugieren que este montículo pudo haberse formado por una serie de depósitos, que al menos en parte pudieron haber estado relacionados con prácticas rituales y ceremonias (Miguez et al. 2012; Miguez et al. 2013; Miguez y Caria 2015).

El extremo sur de la $\mathrm{EM}_{3}$ fue impactado por acción de una pala mecánica en el 2007. La excavación de la pala tuvo una extensión aproximada de $400 \mathrm{~m} 2$, hasta que fue detenida por la aparición de abundantes restos arqueoló- gicos. La profundidad promedio de esta excavación era de unos $30 \mathrm{~cm}$, observándose además algunos pozos de huaqueo que llegaban hasta los $60 \mathrm{~cm}$. La intervención de rescate consistió, principalmente, en la inspección superficial del área impactada, la realización de un croquis de los restos detectados y la recolección superficial de los mismos (Martel et al. 2007). Los materiales registrados fueron los siguientes (ver Figura 4): una estructura de rocas metamórficas (lajas) alineadas y semienterradas en el suelo, con otras piedras asociadas, concentraciones de restos cerámicos, restos óseos humanos asociados a cerámica y a otros huesos de fauna entre los que han reconocido algunos de aves y otros de camélidos (Lama glama sp.), 36 cuentas de collar juntas (elaboradas mayormente en minerales de cobre) procedentes de un loci cercano a la concentración anterior, y algunos artefactos de molienda activos y pasivos (ver Figura 4) (Martel et al. 2007; Miguez y Caria 2015). Tras el rescate, los dueños de la finca mostraron y donaron otro conjunto de materiales culturales procedentes de este mismo sector, que

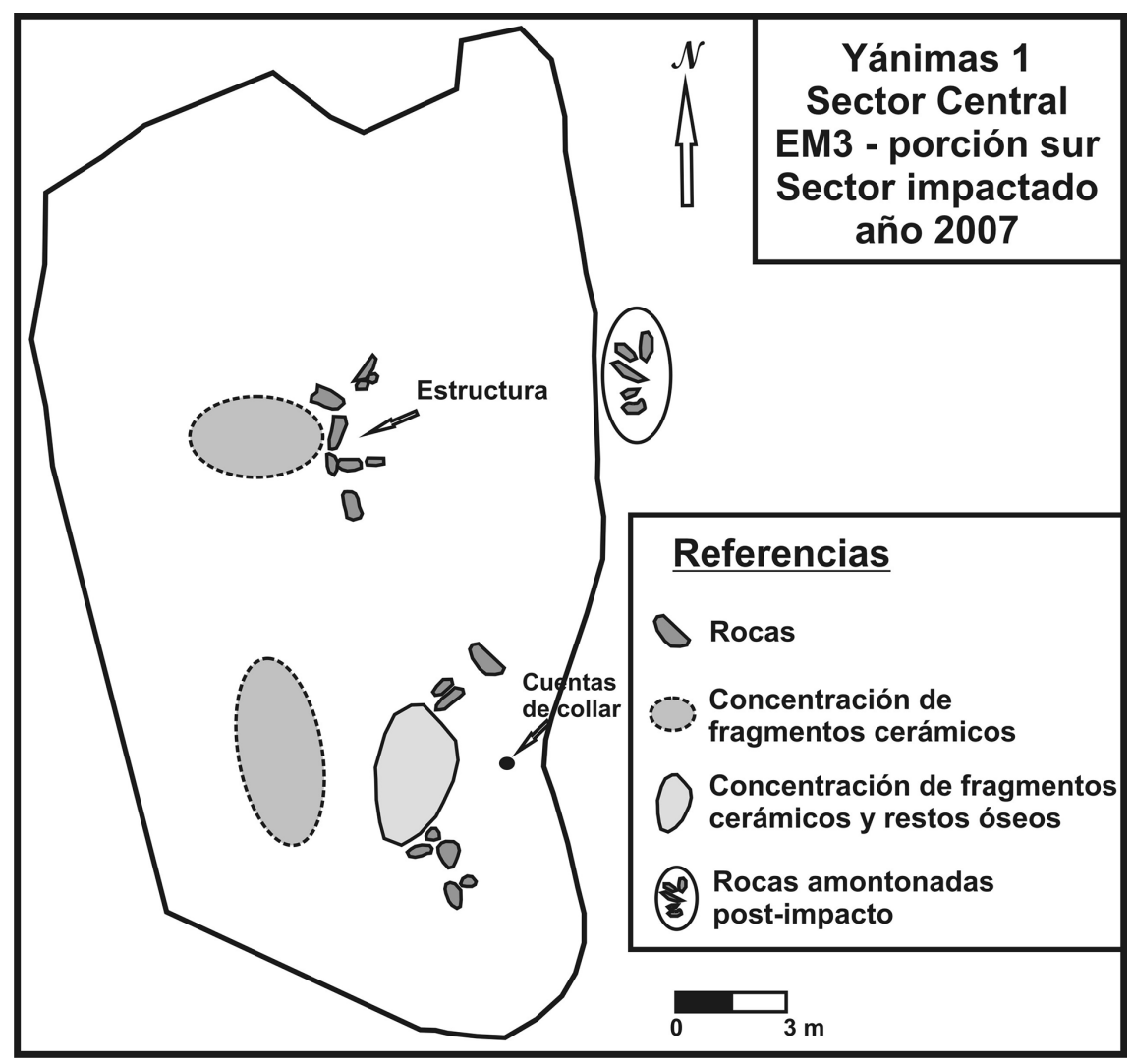

Figura 4. Croquis de los materiales relevados en el sector impactado. 
fueron recolectados al momento de su impacto. Estos restos comprendían abundantes fragmentos cerámicos, una vasija fragmentada, artefactos líticos, restos óseos de animales y humanos y seis fragmentos de pipas (cinco partes de tubos y un hornillo decorado). Este conjunto de restos y sus asociaciones observadas en el terreno nos lleva a plantear que se trataría de un lugar donde se llevaron a cabo múltiples actividades, algunas domésticas y otras posiblemente de carácter ritual, vinculadas con los restos humanos registrados (entierro?) y, tal vez, el uso de pipas (Miguez y Caria 2015).

La alfarería recuperada en estos sectores y en gran parte del sitio, comprende tanto cerámicas finas (con inclusiones de granulometría hasta $1 \mathrm{~mm}$ ) como toscas (con inclusiones hasta $5 \mathrm{~mm}$ ). Dentro de las primeras se reconocieron dos grupos, uno que presenta características tecno-estilísticas (color negro/gris oscuro, superficies pulidas, pasta compacta, decoraciones grabadas/incisas) compatibles con el estilo Ambato Negro Grabado, y otro que se compone de fragmentos de colores rojos/anaranjados tanto en pasta como en superficie (las cuales suelen estar muy alisadas o pulidas), que en ocasiones presentan decoración pintada y/o grabada. Dentro de las cerámicas toscas, además de tiestos no decorados (los más frecuentes), se identificó un grupo que posee atributos (formas de tamaño grande, abundantes inclusiones de mica y cuarzo, superficies alisadas y decoración pintada) en el cual se reconocieron varios fragmentos comparables con el estilo Ambato Tricolor o Alumbrera Tricolor. Los grupos cerámicos descriptos son los más representativos en el sitio (Miguez 2014; Miguez y Caria 2015), aunque se han registrado otros. $\mathrm{Al}$ respecto, cabe mencionar el hallazgo, en una excavación efectuada en la base sur del montículo EMı, de cerámica afín con estilos chacosantiagueños, tales como Averías, asociada a tiestos que se correlacionan con los estilos mencionados anteriormente $e^{7}$. En relación a los estilos mencionados (Ambato Negro Grabado, Alumbrera o Ambato Tricolor y Averías) si bien fueron definidos como característicos de otras áreas (valles orientales y llanuras orientales), son bastante

7 Las definiciones de estos estilos alfareros Ambato Negro Grabado y Ambato Tricolor pueden encontrarse en González (1998: 202-212). Para una definición del Alumbrera Tricolor ver Núñez Regueiro (1998: 175, 182), y para el estilo Averías consultar, por ejemplo, Bonnin y Laguens (2000: 182-183). frecuentes en contextos arqueológicos del piedemonte centro-meridional de Tucumán y en zonas aledañas, de un mismo asentamiento o de ocupaciones diferenciadas tanto espacial como temporalmente (Gómez Augier et al. 2007; Manasse 1997; Pantorrilla y Núñez Regueiro 2006; Rendace et al. 2006; Tartusi y Núñez Regueiro 2003). En el caso de Yánimas I estos estilos se encuentran compartiendo espacios con otras modalidades cerámicas, lo que nos sugiere que en esta zona se dieron interacciones entre diversas comunidades que terminaron por caracterizar los procesos socioculturales en estas selvas del sur de Tucumán (Miguez y Caria 2015).

El conjunto de elevaciones monticulares del Sector Central están delimitando un extenso espacio llano en el centro, que carece de estructuras o divisiones de algún tipo y presenta escasos materiales arqueológicos. Este sector podría haber sido empleado como un espacio abierto de carácter público, de circulación e interacción entre agentes de la misma población para el desarrollo de actividades diversas (Miguez et al. 2013). En los alrededores de la zona del Sector Central o monticular (sectores $\mathrm{O}$, $\mathrm{NO}, \mathrm{N}, \mathrm{NE}$ y E) se registraron en superficie abundantes fragmentos de vasijas, instrumentos de molienda y artefactos líticos tallados, pulidores, restos óseos, piedras, entre otros materiales que sugieren la presencia espacios donde se desarrollaron diversas actividades cotidianas (procesamiento de alimentos, producción y uso de artefactos líticos, utilización de vasijas, consumo de fauna) posiblemente vinculadas a ámbitos residenciales. Asimismo, también se recuperaron algunos fragmentos de pipas y estatuillas en superficie, entre otros elementos que podrían estar sugiriendo el desenvolvimiento de prácticas rituales en estos espacios domésticos (Miguez et al. 2013). En el SN, además, se registró un contexto (de donde procede uno de los fechados) que consiste en restos humanos correspondientes a un entierro directo al menos dos individuos, asociados a otros vestigios, entre los 90-100 cm de profundidad.

\section{Las pipas}

Se registró un total de 12 fragmentos de pipas que corresponderían a la ocupación arriba descrita, todas confeccionadas en cerámica. Diez ejemplares fueron encontradas en el Sector Central (cuatro fueron registras en $\mathrm{EM}_{1}$ y seis en la porción sur de $\mathrm{EM}_{3}$ ) y al menos dos 


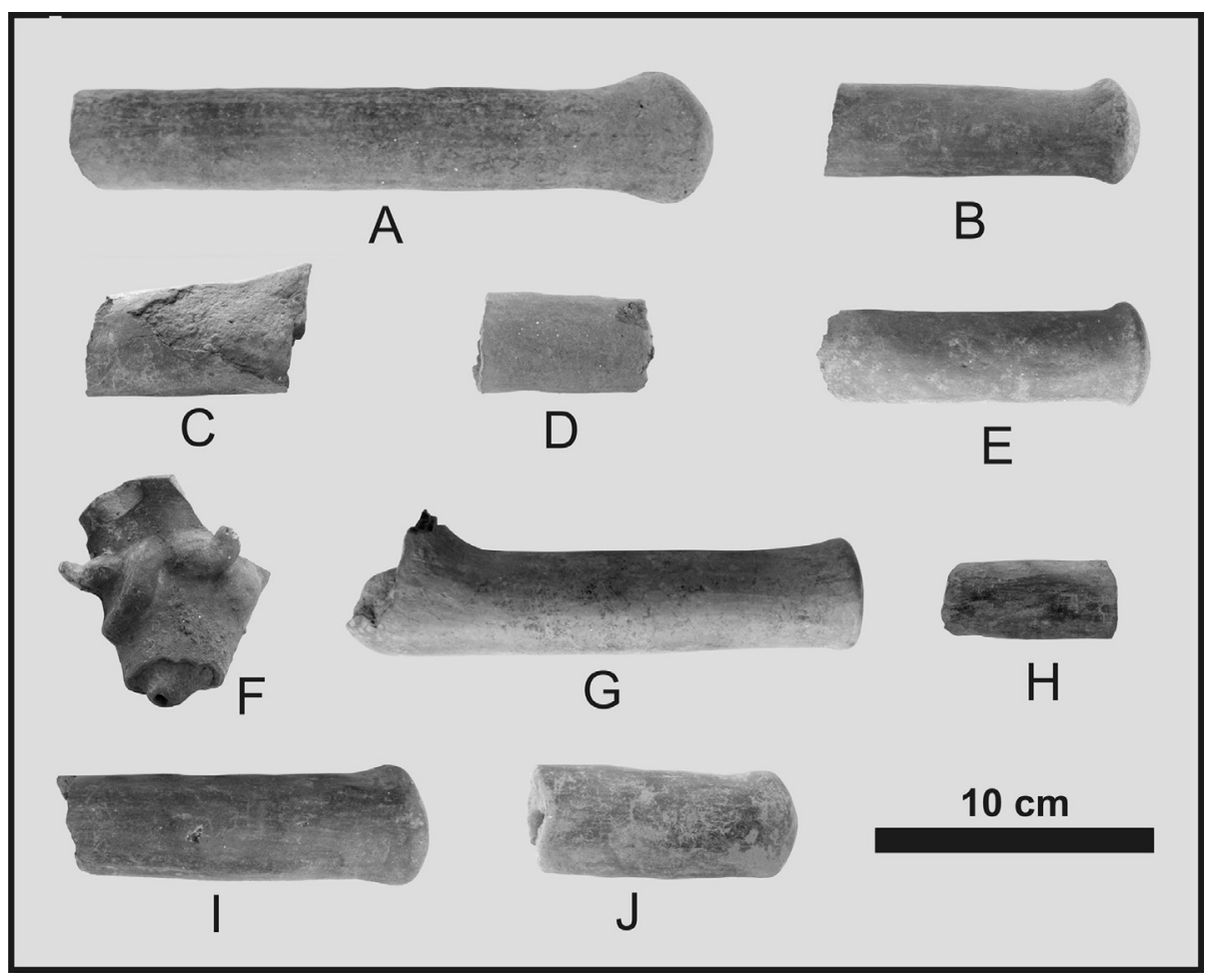

Figura 5. Fragmentos de pipas del sector EM 1: A-D, tubos de aspiración. Fragmentos de pipas del sector EM3: E, G-J, tubos de aspiración. F, hornillo de pipa. Escala: $10 \mathrm{~cm}$.

\begin{tabular}{|c|c|c|c|c|c|}
\hline Pipas & Procedencia & $\begin{array}{c}\text { Medidas } \\
(\mathrm{mm})\end{array}$ & Forma & $\begin{array}{l}\text { Tratamiento } \\
\text { Superficial }\end{array}$ & Huellas de uso \\
\hline $\mathrm{A}$ & $\begin{array}{c}\text { SC - EM1 - Unidad 6 - } \\
\text { Nivel } 5 \text { - Prof: : } 40-50 \mathrm{~cm}\end{array}$ & $\begin{array}{c}\text { L: } 214 / A: 35 \\
\text { E: } 9\end{array}$ & $\begin{array}{l}\text { Fragmento de tubo de forma } \\
\text { cilíndrica, levemente curvado. } \\
\text { Boquilla con terminación curva. }\end{array}$ & Pulido. Pintura negra sobre rojo? & $\begin{array}{l}\text { Areas con des gaste superficial en la } \\
\text { boquilla. Residuos de carbón adheridos en } \\
\text { canal interno. }\end{array}$ \\
\hline $\bar{B}$ & $\begin{array}{c}\text { SC - EM1 - Unidad 6 - } \\
\text { Nivel } 5 \text { - Prof: } 40-50 \mathrm{~cm}\end{array}$ & $\begin{array}{c}\text { L: } 100 / \mathrm{A}: 30 \\
\text { E: } 7\end{array}$ & $\begin{array}{l}\text { Fragmento de tubo de forma } \\
\text { cilíndrica. Boquilla con } \\
\text { terminación curva. }\end{array}$ & Pulido. & $\begin{array}{l}\text { Areas con desgaste superficial y mancha } \\
\text { oscura alrededor del orificio de la boquilla. } \\
\text { Residuos de carbón en canal interno. }\end{array}$ \\
\hline $\mathrm{C}$ & $\begin{array}{l}\text { SC - EM1 - Unidad 1- } \\
\text { Nivel 10-Prof.:90-100 cm }\end{array}$ & $\begin{array}{l}\text { L: } 66 \text { / A: } 41 \\
\text { E: } 10\end{array}$ & $\begin{array}{c}\text { Fragmento de tubo de forma } \\
\text { ovalada. }\end{array}$ & Alisado & Residuos de carbón en canal interno. \\
\hline$\overline{\mathrm{D}}$ & $\begin{array}{l}\text { SC - EM1 - Unidad 8 - } \\
\text { Nivel } 5 \text { - Prof.: } 40-50 \mathrm{~cm}\end{array}$ & $\begin{array}{l}\text { L: } 55 / A: 32 \\
\text { E: } 9,5\end{array}$ & $\begin{array}{l}\text { Fragmento de tubo de forma } \\
\text { cilíndrica. }\end{array}$ & Alisado. & Residuos de carbón en canal interno. \\
\hline $\bar{E}$ & SC-EM3 - Superficie & $\begin{array}{c}\text { L: } 103 / \mathrm{A}: 29 \\
\text { E: } 8\end{array}$ & $\begin{array}{l}\text { Fragmento de tubo de forma } \\
\text { cilíndrica. Boquilla con } \\
\text { terminación curva. }\end{array}$ & Pulido. & $\begin{array}{l}\text { Areas con des gaste superficial en la } \\
\text { boquilla. Residuos de carbón adheridos en } \\
\text { canal interno y en sectores externos. }\end{array}$ \\
\hline F & SC-EM3 - Superficie & $\begin{array}{c}\text { L: } 74,5 / \mathrm{A}: 55 \\
\mathrm{E}:-\end{array}$ & $\begin{array}{l}\text { Fragmento de hornillo de forma } \\
\text { cónica, con bordes evertidos. }\end{array}$ & $\begin{array}{l}\text { Alisado. Decorado con dos } \\
\text { apéndices modelados y pintura } \\
\text { roja (en bordes superior e } \\
\text { inferior) sobre fondo negro. }\end{array}$ & $\begin{array}{c}\text { Escasos residuos de carbón adheridos a la } \\
\text { superficie interna del hornillo. }\end{array}$ \\
\hline $\mathrm{G}$ & SC - EM3 - Superficie & $\begin{array}{c}\text { L: } 156 / A: 34 \\
\text { E: } 4\end{array}$ & $\begin{array}{l}\text { Tubo completo de forma } \\
\text { cilíndrica. Boquilla con } \\
\text { terminación recta. }\end{array}$ & Pulido. & $\begin{array}{l}\text { Areas con desgaste superficial en la } \\
\text { boquilla. Residuos de carbón en canal } \\
\text { interno. }\end{array}$ \\
\hline $\mathrm{H}$ & SC-EM3 - Superficie & $\begin{array}{l}\text { L: } 52 / A: 27 \\
\text { E: } 7\end{array}$ & $\begin{array}{l}\text { Fragmento de tubo de forma } \\
\text { ovalada. }\end{array}$ & Alisado fino. & Residuos de carbón en canal interno. \\
\hline I & SC-EM3 - Superficie & $\begin{array}{c}\text { L: } 120 / A: 36 \\
\text { E: } 5\end{array}$ & $\begin{array}{l}\text { Fragmento de tubo de forma } \\
\text { cilíndrica. Boquilla con } \\
\text { terminación curva. }\end{array}$ & Pulido. Pintura roja y negra? & Residuos de carbón en canal interno. \\
\hline $\mathbf{J}$ & SC - EM3 - Superficie & $\begin{array}{c}\text { L: } 86 / \mathrm{A}: 37 \\
\text { E: } 12\end{array}$ & $\begin{array}{l}\text { Fragmento de tubo de forma de } \\
\text { cilíndrica irregular. Boquilla con } \\
\text { terminación curva. }\end{array}$ & Alisado fino. & Residuos de carbón en canal interno. \\
\hline
\end{tabular}

Tabla 1. Caracterización formal y huellas de uso observadas en las pipas. 
fragmentos de tubos pudieron identificarse para el Sector Norte, estos últimos obtenidos mediante recolección superficial. Para este trabajo fueron seleccionadas las pipas del SC (Figuras $5 \mathrm{~A}-\mathrm{J}$ ), dado que provienen de contextos mejor relevados y, en su mayoría, preservaban sus contenidos internos (sedimentos y residuos de carbón) casi inalterados. En la Tabla 1 se detallan sus medidas y características formales, y se describen las huellas de uso observadas. Al respecto, resulta interesante que además de los residuos de carbón observados en las paredes internas de los tubos, se han podido identificar otras huellas de uso tales como sectores desgastados y manchas (de grasitud?) en las boquillas, producidas muy probablemente por el contacto recurrente de los labios de los fumadores con las pipas.

Resulta difícil determinar con precisión el estilo al que corresponderían estas pipas, dado que la mayoría corresponden a partes de los tubos de inhalación/boquillas. Si bien se intentó ensamblar estas partes (por ejemplo, los fragmentos de tubos con el hornillo), se obtuvieron resultados negativos. No obstante, a partir del hornillo y el tubo/boquilla completo registrado en $\mathrm{EM}_{3}$ (Figuras. $5 \mathrm{~F}$ y $G$ respectivamente), se infiere que al menos parte de las pipas utilizadas en este sitio a fines del primer milenio DC fueron acodadas o de hornillo vertical. Por la forma de los tubos y boquillas y, asimismo, por las características formales y decoración del hornillo, se podría decir que son comparables con las pipas registradas en el valle de Ambato y zonas aledañas (ver González 1998: 89-91).

\section{MATERIALES Y MÉTODOS}

\section{Material botánico actual de referencia}

Para la confección del material botánico actual de referencia se procedió a una búsqueda de bibliografía sobre el uso de enteógenos en el NOA, y se consultó al Biólogo Omar Varela, del Instituto de Ecología de la Fundación Miguel Lillo (Tucumán). A partir de esto, las especies seleccionadas fueron: cebil -Anadenanthera colubrina var. cebil (Griseb.) Altschul- (semilla), tabaco -Nicotiana spp.(hoja), y tabaquillo-Nicotiana longuiflora- (hoja). A su vez con el fin de aumentar la colección de referencia y considerando los estudios realizados por Singh (2003), se incluyó el cactus de San Pedro -Echinopsis pachanoi- (tallo). La determinación de las especies y de los diferentes órga- nos específicos en cada planta, se hizo sobre la base de su dispersión geográfica y los antecedentes bibliográficos (estudios arqueológicos, etnohistóricos y etnográficos) que se encontraron para el área (Pérez Gollán y Gordillo 1993, 1994; Pochettino y Capparelli 2004; entre otros).

Para la confección de los preparados de referencia del material botánico actual, las muestras fueron sometidas a dos protocolos paralelos: a) Calcinación en mufla y b) Diafanización en $\mathrm{H}_{2} \mathrm{O}_{2}+$ calor. El primer protocolo consistió en separar muestras de las diferentes especies seleccionadas (cebil - semilla-, tabaco -hoja-, tabaquillo -hoja- y cactus de San Pedro -tallo-) en seco que fueron colocadas en cápsulas de Petri, y luego llevadas a una mufla hasta que esta alcanzara los $550^{\circ} \mathrm{C}$ por un lapso de 2 horas. Posteriormente, se cambiaron a cápsulas más pequeñas y se colocaron unas gotas de ácido acético para eliminar cualquier vestigio de restos orgánicos, sin alterar la presencia posible de oxalatos de calcio o calcifitolitos (Coil et al. 2003). En cuanto al protocolo de Diafanización, al igual que el protocolo anterior, se procedió a la separación de muestras de las especies previamente seleccionadas en húmedo, que luego se colocaron sobre una plancha calefactora y en agua oxigenada de 100 vol., eliminando así el tejido orgánico. Seguidamente, las muestras fueron llevadas a una campana de extracción de gases y lavadas con agua destilada, revolviendo con una cuchara de metal, siendo finalmente depositadas en nuevas cápsulas de Petri. Además se realizaron cortes delgados de San Pedro (Echinopsis pachanoi), los cuales fueron realizados en el INSIBIO (Instituto Superior de Investigaciones Biológicas, Tucumán).

La observación de los preparados se realizó bajo microscopio petrográfico (Zeiss Axioscope), que cuenta con lente micrograduado, polarizador y cámara incorporada. Se trabajó 10 campos con aumentos de 200x. Se fotografió en campo claro y oscuro (con cuña de cuarzo).

\section{Material arqueológico}

El material arqueológico analizado está compuesto por muestras de contenidos (residuos adheridos) procedentes de 10 fragmentos de pipas cerámicas recuperadas en dos montículos (EM 1 y EM3). La obtención se realizó mediante el raspado del material presente en los fragmentos de las pipas (tubos de aspiración, y hornillo en el 
caso de la muestra F del sector EM 3$)^{8}$. El material obtenido fue colocado en cápsulas de Petri y sometido a tres protocolos: a) Clásico (Pochettino y Capparelli 2004): consistió en colocar agua oxigenada (100 vol. al 30\%) a las muestras, dejándolas durante dos días en este medio. Luego se colocaron en tubos de ensayos con agua destilada, los cuales fueron llevados a la centrífuga (3000 revoluciones por minuto) y después se retiró el líquido sobrante de los tubos quedando el material restante depositado en el fondo; b) Tamizado, se empleó un tamiz de 75 micras sobre las muestras de cada pipa, depositándolas en cápsulas de Petri (Canti 1997); y c) el protocolo de extracción directa de residuos (Loy 1994; Korstanje y Babot 2007), donde el material obtenido del raspaje de las pipas fue colocado en cápsulas de Petri, sin añadir agua oxigenada, con la finalidad de recuperar componentes orgánicos tales como almidones.

Los preparados se observaron y fotografiaron con microscopio petrográfico. Se trabajaron 20 campos con aumentos de 200x. Se identificaron, midieron, describieron los atributos relevantes de los microvestigios presentes en las muestras (silicofitolitos, almidones, calcifitolitos). La descripción morfológica y atributos de los silicofitolitos se basó en el International Code for Phytolith Nomenclature 1.o. (Madella et al. 2005), se utilizaron los criterios de formas tridimensionales y bidimensionales; y aspectos de la superficie. En el caso de los almidones se tuvo en cuenta la presencia de rasgos diagnósticos, como for- ma, tamaño y birrefrigencia. Asimismo, lo observado fue comparado con el material botánico actual de referencia, y con publicaciones especializadas (Babot 2004; Korstanje y Babot 2007; entre otros).

\section{RESULTADOS}

En las muestras arqueológicas procedentes del sector $\mathrm{EM}_{1}$ se pudo observar que tanto los almidones ( $n=4.429)$, silicofitolitos $(n=93)$, microcarbones, anillos de celulosa $(n=42)$ y, en menor proporción, calcifitolitos $(n=3)$, se encuentran representados en la mayoría de las muestras (ver Tabla 2). También se observa una abundancia de almidones sobre otros microvestigios, los almidones identificados se atribuyen como característicos de la hoja de tabaco (Nicotiana spp) ${ }^{9}$ caracterizados por Babot (2004) como granos ovales, con cruz de extinción visible y central con cuatro brazos visibles. (Figuras. 6 A-B). En menor proporción se han reconocido otros almidones asignables a cebil [Anadenantheracolubrinavar. cebil (Griseb.) Altschul], los cuales se distinguen por ser de

8 Cabe aclarar que los instrumentos de laboratorio empleados para la toma de muestras de sedimentos fueron esterilizados y enjuagados con agua oxigenada entre muestra y muestra, con el fin de evitar contaminación cruzada.

9 Se ha preferido usar la denominación Nicotiana spp. hasta la realización de estudios complementarios que permitan determinar con certeza las especies presentes en las muestras.

\begin{tabular}{|c|c|c|c|c|c|}
\hline \multirow{2}{*}{ Pipas } & Microvestigios & & & & \\
\hline & Silicofitolitos & Calcifitolitos & Granos de Almidón & Anillos de celulosa & Microcarbones \\
\hline $\mathrm{Al}_{1}$ & 22 & & 27 & 4 & \\
\hline $\mathrm{A}_{2}$ & 4 & & & & $\mathrm{X}$ \\
\hline $\mathrm{A}_{3}$ & 3 & & 874 & 1 & $\mathrm{X}$ \\
\hline $\mathrm{B}_{1}$ & 21 & & 387 & 8 & $\mathrm{X}$ \\
\hline $\mathrm{B}_{2}$ & 14 & & 533 & & $\mathrm{X}$ \\
\hline $\mathrm{B}_{3}$ & 16 & & 916 & & $\mathrm{X}$ \\
\hline $\mathrm{Cl}_{1}$ & 3 & & 413 & 2 & $\mathrm{X}$ \\
\hline $\mathrm{C}_{2}$ & 1 & & 114 & 6 & $\mathrm{X}$ \\
\hline $\mathrm{C}_{3}$ & 3 & & 93 & & $\mathrm{X}$ \\
\hline$D_{1}$ & & 3 & & & $\mathrm{X}$ \\
\hline $\mathrm{D}_{2}$ & & & 78 & 1 & $\mathrm{X}$ \\
\hline $\mathrm{D}_{3}$ & 6 & & 994 & 23 & $\mathrm{X}$ \\
\hline Total & 93 & 3 & 4.429 & 42 & \\
\hline
\end{tabular}

Tabla 2. Síntesis de resultados y detalle de cantidades según tipos de microfósiles recuperados del tubo y/o boquillas de las pipas analizadas del sector EMı. En el caso de los microcarbones no se contabilizaron cantidades sino presencia/ausencia en las diferentes muestras. 

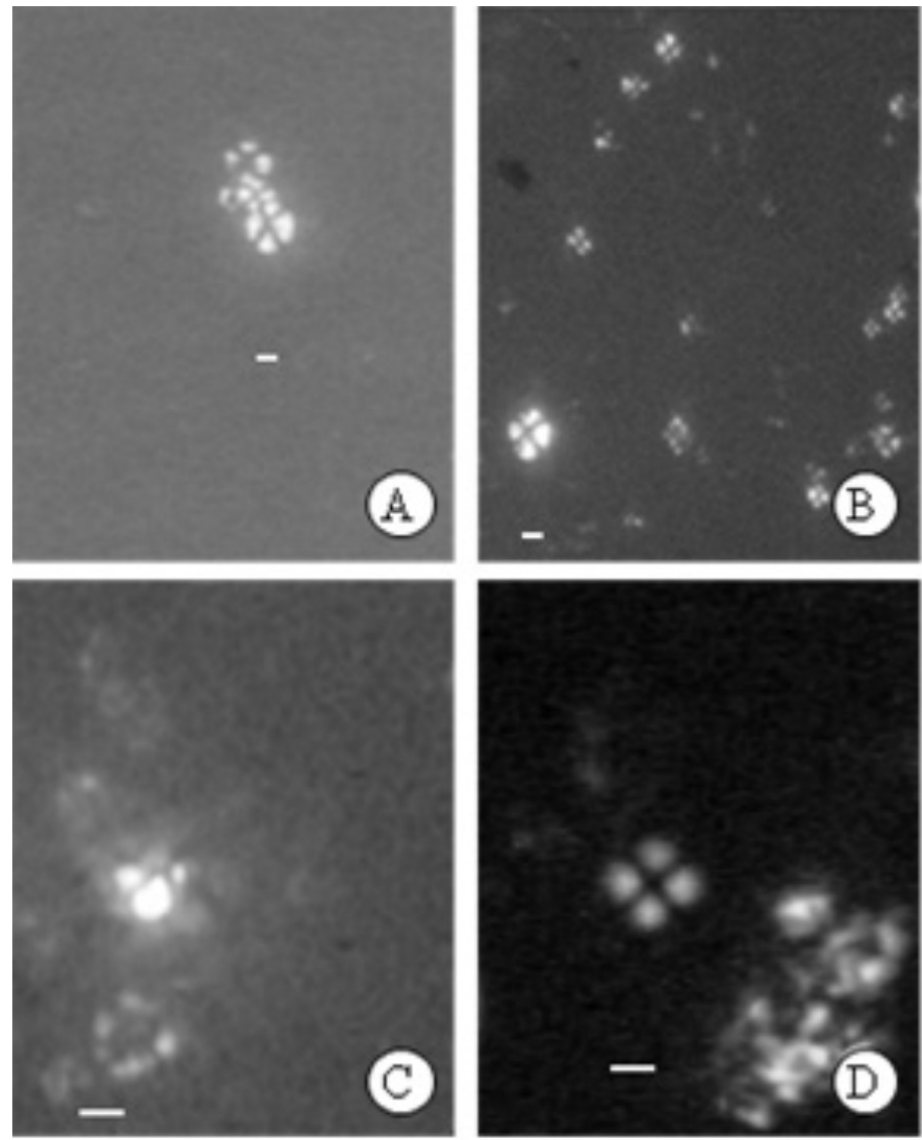

Figura 6. Granos de almidón recuperados sector EMı. A, muestra Bı granos de almidón afines a tabaco (Nicotiana spp.) B, muestra D3 afines a Nicotiana spp. C, grano de almidón Anadenanthera colubrina var. cebil. (Griseb.)

Altschul. D, muestra D3 grano de almidón indeterminado. Escala: $5 \mu \mathrm{m}$.

grano más alargado -no tan esférico como el del tabaco-y poseer una depresión circular, oval en el centro con forma similar a la del contorno del grano, y con cuatro brazos visibles intersectados en la depresión oscura (Figura 6 C). Además se registró un conjunto de granos de almidones indeterminados en los que no se ha encontrado su correlato con las colecciones de referencia (Figura 6 D). A su vez, es necesario señalar la presencia de almidones en las muestras tratadas con el protocolo Clásico (a), a excepción de la muestra Di. Si bien los almidones pueden sobrevivir a tratamientos que impliquen agua oxigenada, como es el caso que se presenta en nuestras muestras de referencia, el número elevado de almidones presentes en las mismas nos llevan a plantear la corroboración de estos resultados con futuros estudios complementarios. Descartamos cualquier tipo de contaminación, dado que además se trata de un tipo de almidón particular [tabaco
(Nicotiana spp)], que no es manipulado frecuentemente en las casas ni laboratorios.

Los residuos analizados en las muestras de este sector indican una mayor presencia y variedad de silicofitolitos en las muestras A y B, correspondiendo estas muestras a la misma unidad de procedencia (Unidad 6, nivel 5) (Figuras $7 \mathrm{~A}-\mathrm{E}$ ). Se observaron morfotipos semejantes a los que Pearsall y Dinan (1992) describen como silicofitolitos representativos de la familia Poaceae en la muestra $\mathrm{Al}$ (silicofitolitos elongados con bordes aserrados) y en la muestra $\mathrm{C}_{2}$ (silicofitolitos bilobulados) (Figuras $7 \mathrm{~A} \mathrm{y}$ F), la identificación de morfotipos de esta familia podría estar indicando la presencia de gramíneas. Sumado a esto, en la muestra B2 se identificó un fitolito bulliforme (Figura $7 \mathrm{C}$ ), que si bien no ha sido posible determinar especie o familia, ayuda a corroborar el empleo de ma- 

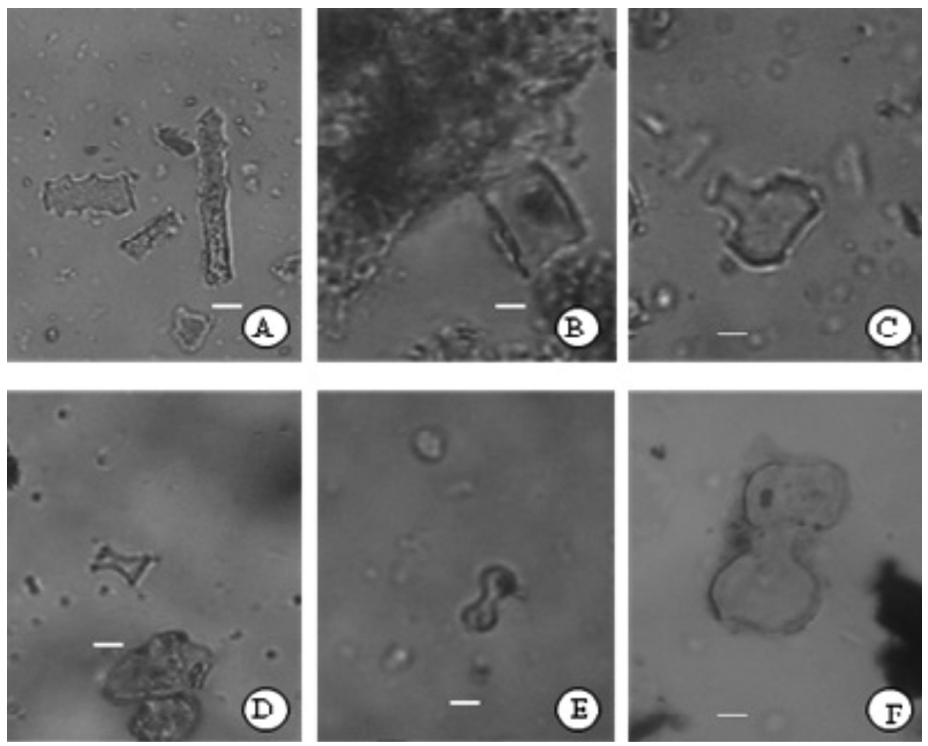

Figura 7. Silicofitolitos recuperados en las pipas del sector EMı A, muestra $A_{1}$ silicofitolitos elongados con bordes aserrados. B, muestra B1 silicofitolito cuadrado. C, muestra B2 silicofitolito bulliforme, base recta y hombros redondeados. D, muestra B1 silicofitolito en forma de cono truncado. E, muestra B1 silicofitolito bilobulado. F, muestra C2, silicofitolito bilobulado representativo de la familia Poaceae. Escala $5 \mu \mathrm{m}$.
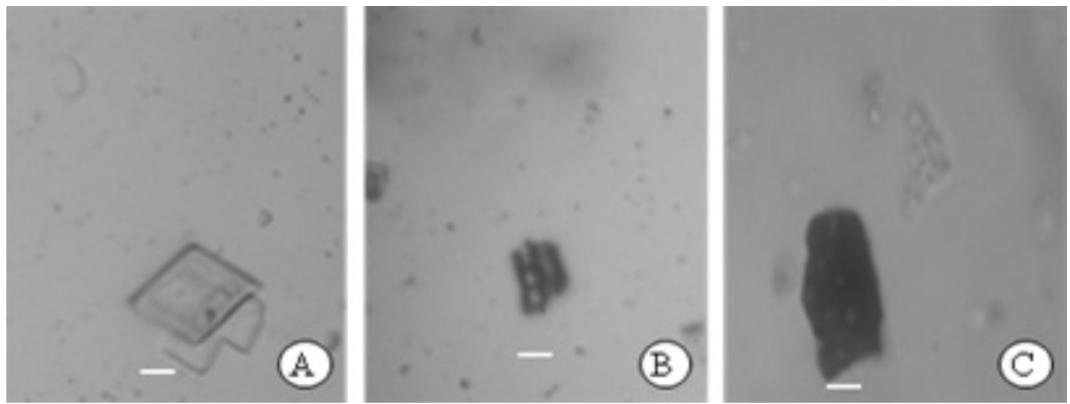

Figura 8. Microvestigios pipas del sector EMı A, muestra D1,calcifitolito romboide afín a Anadenanthera colubrina var. cebil (Griseb.) Altschul. B-C, muestra $\mathrm{C}_{1}$, placas perforadas. Escala $5 \mu \mathrm{m}$.

terial foliáceo en las pipas. En el caso de los calcifitolitos, tomando como base los cristales de oxalato de calcio identificados en la muestra M8 de cebil perteneciente a la colección de referencia ${ }^{10}$, se registraron en la muestra Di calcifitolitos afín a Anadenanthera colubrina var. cebil (Griseb) Altschul (Figura 8 A). Además, en la muestra $\mathrm{C}_{1}$ se observaron dos placas perforadas posiblemente por su morfología correspondientes a dicotiledóneas (Figuras 8 B y C), y en la muestra Di se observó una espora, que a futuro se deberá determinar el origen de su ocurrencia (sin descartar diferentes factores actuantes como las fuentes de contaminación post-depositacional).

En el sector $\mathrm{EM}_{3}$, los microvestigios detectados en las muestras revelan una amplia variedad de formas y distintas procedencias anatómicas (ver Tabla 3). Los granos de almidón identificados, en su mayoría, estarían correspondiendo por su morfología a almidones afines a Nicotiana spp., sensu Babot (2004) (Figura 9 A-B). Pero también se identificaron en menor proporción almidones que corresponderían a los descriptos para el cebil [Anadenanthera colubrina var. cebil (Griseb.) Altschul]. En cuanto a los fitolitos detectados se destacan las formas bilobulada y cuadrangular. Los silicofitolitos bilobulados se encon-

10 Estos calcifitolitos se caracterizan por tener muy buena fractura y por brillar con luz polarizada en campo oscuro (Babot 2004: 102). 
Valeria B. Martín Silva, Gabriel E. Miguez y M. Alejandra Korstanje

\begin{tabular}{|c|c|c|c|c|c|}
\hline \multirow[b]{2}{*}{ Pipas } & \multicolumn{2}{|l|}{ Microvestigios } & \multirow[b]{2}{*}{$\begin{array}{l}\text { Granos de } \\
\text { Almidón }\end{array}$} & \multirow[b]{2}{*}{$\begin{array}{c}\text { Anillos de } \\
\text { celulosa }\end{array}$} & \multirow[b]{2}{*}{ Microcarbones } \\
\hline & Silicofitolitos & Calcifitolitos & & & \\
\hline E1 & & & 1 & & $\mathrm{X}$ \\
\hline $\mathrm{E}_{2}$ & 4 & & 39 & 6 & $\mathrm{x}$ \\
\hline $\mathrm{E}_{3}$ & 2 & 3 & & & $\mathrm{X}$ \\
\hline $\mathrm{F}_{1}$ & 7 & & 37 & 8 & $\mathrm{x}$ \\
\hline $\mathrm{F}_{2}$ & 5 & & 9 & & $\mathrm{x}$ \\
\hline $\mathrm{F}_{3}$ & 5 & & 9 & & $\mathrm{x}$ \\
\hline $\mathrm{Gl}$ & 11 & & 159 & & $\mathrm{x}$ \\
\hline $\mathrm{G}_{2}$ & & & 5 & & $\mathrm{x}$ \\
\hline $\mathrm{G}_{3}$ & 1 & & 328 & 25 & $\mathrm{x}$ \\
\hline $\mathrm{H}_{1}$ & & & 65 & & $\mathrm{x}$ \\
\hline $\mathrm{H}_{2}$ & 1 & 20 & 689 & 25 & $\mathrm{x}$ \\
\hline $\mathrm{H}_{3}$ & 3 & & 1 & & $\mathrm{X}$ \\
\hline $\mathrm{I}_{1}$ & 2 & & 1 & & $\mathrm{X}$ \\
\hline $\mathrm{I}_{2}$ & 2 & & & & $\mathrm{x}$ \\
\hline $\mathrm{I}_{3}$ & 2 & & 19 & & $\mathrm{x}$ \\
\hline $\mathrm{J}_{1}$ & 2 & & 3 & & $\mathrm{x}$ \\
\hline $\mathrm{J}_{2}$ & 2 & & 3 & & $\mathrm{X}$ \\
\hline $\mathrm{J}_{3}$ & 3 & & & & $\mathrm{X}$ \\
\hline
\end{tabular}

Tabla 3. Síntesis de resultados y detalle de cantidades según tipos de microfósiles recuperados de recuperados del tubo y/o boquillas (Muestras E, G-J) y del hornillo (Muestra F) de las pipas analizadas del sector $\mathrm{EM}_{3}$.

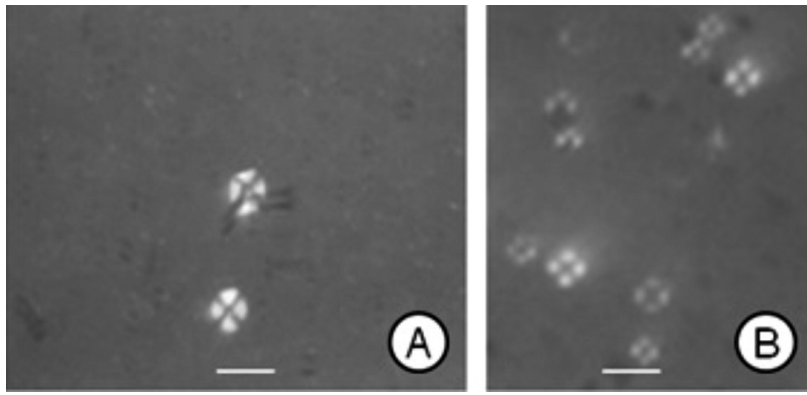

Figura 9. Granos de almidón recuperados de las pipas del sector $\mathrm{EM}_{3}$, afines a tabaco (Nicotiana spp.) A, muestra G1. B, muestra G2. Escala: $5 \mu \mathrm{m}$.
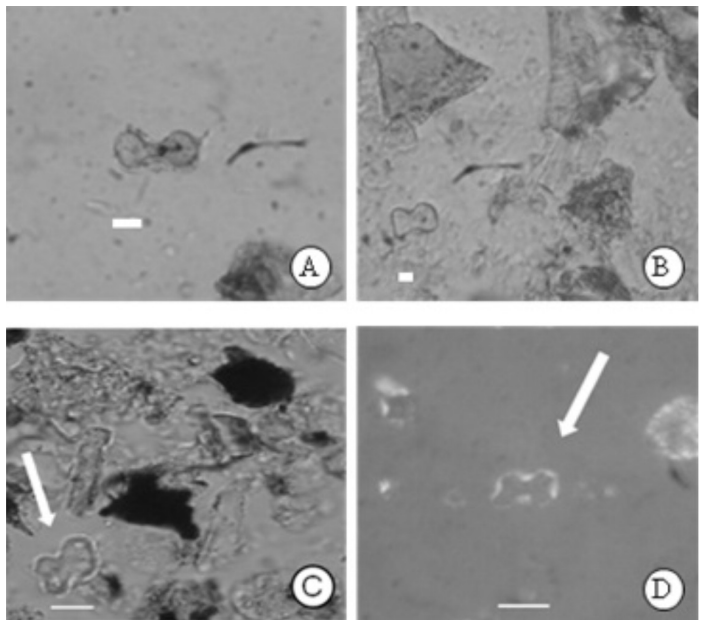

Figura 10. Silicofitolitos recuperados de las pipas del sector $\mathrm{EM}_{3}$.A, silicofitolito bilobulado de centro corto y final convexo. B, silicofitolito bilobulado de centro corto y final cóncavo. C-D, silicofitolitos bilobulados representativos de la familia Poaceae. 
traron presentes en casi todas las muestras arqueológicas de este sector (Figura 10 A-B). En las muestras $\mathrm{E}_{3}, \mathrm{Fl}$, $\mathrm{F}_{2}, \mathrm{~F}_{3}, \mathrm{G} 1, \mathrm{G} 2, \mathrm{G}_{3}$, I3 y J1 se han observado, al igual que en muestras del sector EM1, silicofitolitos representativos de la familia Poaceae (Figuras $10 \mathrm{C}-\mathrm{D}$ ), indicando la posible presencia de gramíneas.

Los calcifitolitos sólo se encontraron en las muestras $\mathrm{E}_{3}, \mathrm{H}_{2}$ y I3. La morfología de los calcifitolitos que se observan en las muestras se atribuyen afines a aquellos del cebil [Anadenanthera colubrina var. cebil (Griseb.) Altschul] (Figuras 11 A-B). En el caso de la muestra H2, presentan forma bien definida tipo romboide, con un tamaño de 7,5 x 7,5 micras en 4ox. Asimismo, la muestra $\mathrm{H}_{2}$ se registró una abundante cantidad de calcifitolitos (20) por sobre las demás muestras, siendo esta muestra la más representativa de este tipo de microvestigio.
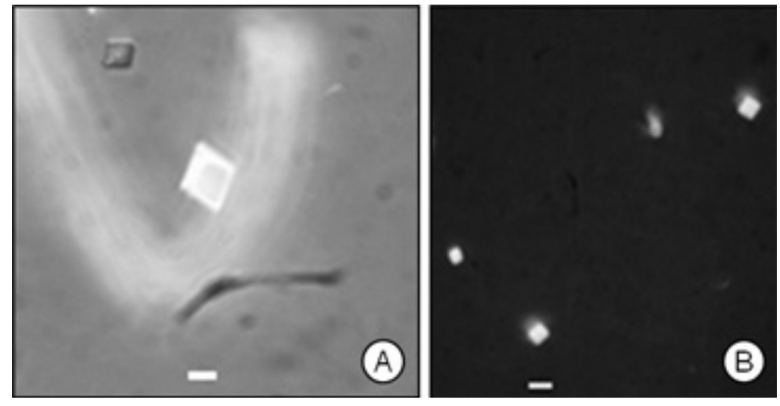

Figura 11. Calcifitolitos recuperados en el sector $\mathrm{EM}_{3}$, correspondientes a cebil [Anadenanthera colubrina var. cebil (Griseb.) Altschul].A, muestra $\mathrm{E}_{3} \mathbf{B}$, muestra $\mathrm{H}_{2}$. Escala: $5 \mu \mathrm{m}$.

\section{DISCUSIÓN Y CONCLUSIONES}

Los protocolos de laboratorio y, en general, la metodología aplicada a las muestras de los residuos adheridos de las pipas de los montículos $\mathrm{EM}_{1}$ y $\mathrm{EM}_{3}$, han permitido la identificación de una importante variedad de microvestigios que proceden de varias especies de plantas. Por un lado, la presencia de silicofitolitos correspondientes a la familia Poaceae (gramíneas) podría dar cuenta del posible uso de estas plantas como material combustible o, tal vez, de su empleo en conjunto con plantas enteógenas como catalizadoras de la reacción psicoactiva. Sin embargo, su ocurrencia también puede deberse a que el contenido de las pipas haya experimentado algún ingreso de los residuos que forman parte de la matriz en la que estaban depositados, dado que las gramíneas son comunes en ellas (Quiroz et al. 2012). En este sentido, y dada la inespecificidad de dichas gramíneas, queda como interrogante hasta que las metodologías de distinción sean de grano más fino.

A su vez, se han podido identificar en los residuos de las pipas granos de almidón afines a Nicotiana spp. El tamaño y forma de los granos, la cruz de extinción visible y central con cuatro vasos brazos visibles (hilado centra- do), han sido los rasgos que permitieron otorgarle esta posible asignación taxonómica. Un dato a destacar es la observación reiterada, tanto en muestras de EMi como de $\mathrm{EM}_{3}$, de almidones asimilables al género Nicotiana spp. en conjunción con almidones y calcifitolitos correspondientes a cebil [Anadenanthera colubrina var. cebil (Griseb.) Altschul]. Si bien es posible que el registro de estas especies pueda ser el resultado de sesiones fumatorias donde se hayan utilizado una u otra planta en los mismos artefactos, la presencia conjunta de ambas plantea también la posibilidad de su utilización en forma simultánea, vale decir, que se hayan fumado mezclados (tabaco + cebil). La preparación del tabaco en combinación con agentes psicotrópicos más activos, como por ejemplo el cebil, es una práctica frecuente en comunidades etnográficas sudamericanas (Califano 1976; Wilbert 1987; Schultes y Hoffman 2000). Al respecto, Califano (1976: 47) señala que en grupos Mataco del río Pilcomayo ha registrado el consumo de una mezcla de tabaco con polvo de semillas de cebil en pipas tubulares de madera. En contextos arqueológicos, además de las mencionadas pipas de piedra de Huachichocana III (Tumbaya, puna de Jujuy), esta asociación de enteógenos ha sido registrada recientemente, en ejemplares de cerámica procedentes de sectores de la vertiente oriental andina, tal como sucede 
en Yánimas 1. Este es el caso de dos ejemplares del valle del río San Francisco (Jujuy), que han sido ubicados aproximadamente entre 2200-1800 AP; como también el de una pipa completa proveniente de la localidad de La Candelaria (Salta) y otro fragmento recuperado en estratigrafía en el sitio El Taco (Ancasti, Catamarca), este último procedente de un estrato que ha sido fechado en 1390 $\pm 70 \mathrm{AP}$ (Lema et al. 2015).

Por otra parte, el registro de microvestigios que corresponderían a estas plantas psicoactivas nos permite realizar algunos comentarios respecto a las estrategias de obtención de las mismas en relación a su distribución biogeográfica. Por un lado, la identificación del cebil [Anadenanthera colubrina var. cebil (Griseb) Altschul]en las muestras analizadas sería concordante con la abundante presencia de este árbol en el área de estudio, en ocasiones formando bosques monoespecíficos, algunos de los cuales se han observado en lugares muy cercanos al sitio (unos de ellos a poco más dos $\mathrm{km}$ en línea recta). Por ello se sugiere que el cebil se habría obtenido en prácticas de recolección llevadas a cabo en las cercanías a esta ocupación prehispánica. En lo que respecta al tabaco (Nicotiana spp.), el nivel taxonómico alcanzado en este estudio no nos permite realizar observaciones concluyentes sobre las posibles formas de obtención en el pasado. La realización de estudios complementarios nos permitirá determinar con certeza las especies presentes en la muestra ${ }^{11}$ y evaluar su distribución biogeográfica en relación a la ubicación del sitio Yánimas 1. En principio no se descarta su origen local. Pero también es probable que la/s especie/s utilizada/s por parte de la comunidad que habitó este sitio a fines del primer milenio DC no sean locales, ya que en el piedemonte meridional de Tucumán no se registran especies silvestres de este género (si bien actualmente se cultiva $N$. tabacum para su explotación comercial). En caso de confirmarse esta hipótesis, podrían plantearse dos alternativas, que no serían mutua-

$11 \mathrm{Al}$ respecto, en la actualidad un grupo de investigadoras chilenas está avanzando en una mejor identificación de los microvestigios por tipo de especie de nicotiana (Quiroz 2014).

12 Cabe acotar que en otras áreas no tan alejadas de este sitio, como en los valles orientales de Catamarca, crecen otras especies del género Nicotiana (N. longiflora, N. paa) (Clérici et al. 2012; Rosso y Scarpa 2011; http://www2.darwin.edu.ar/Proyectos/FloraArgentina/BuscarEspecies.asp, Consultado Noviembre 2015). mente excluyentes. Por un lado, es posible que ciertos individuos de esta comunidad hayan realizado extensos viajes hacia las zonas donde estas plantas crecen naturalmente. Por ejemplo, Scarpa y Rosso (2011) señalan la presencia de Nicotiana paa (Mart. Crov.) en el norte de Tucumán y en el occidente Santiago del Estero, áreas relativamente cercanas a Yánimas $1^{12}$. Al respecto, es interesante señalar que, entre los grupos chaqueños, se han registrado viajes que cubren largas distancias ("decenas de kilómetros") para la recolección de estas plantas (Rosso y Scarpa 2011:399). Otra alternativa podría ser que se hayan empleado redes de tráfico e intercambio para su obtención, y su consecuente uso como un elemento articulador en el establecimiento de relaciones de interacción interregionales. Como ya hemos señalado, algunos indicadores de estos procesos de interacción pueden observarse en la variabilidad de estilos cerámicos presentes en este sitio, algunos de ellos típicamente representados en la llanura tucumano-santiagueña (Averías).

A escala del sitio, se considera pertinente aquí discutir la relación existente entre las pipas y los contextos de procedencia. Los espacios donde fueron encontrados los artefactos estudiados forman parte de elevaciones monticulares con distintas características. Por un lado se hallaron cuatro fragmentos de tubos en EMr, montículo que por su forma, ubicación y por diversas evidencias registradas en su interior (materiales culturales y sedimentos) ha sido considerado como un espacio monumentalizado de alto carácter simbólico, que tal vez estuvo vinculado a experiencias y prácticas rituales de tipo chamánicas o diversas ceremonias, cuyos materiales pudieron haber contribuido a su formación por medio de depósitos u ofrendas (Miguez et al. 2012; Miguez y Caria 2015). En el caso del nivel 5 (unidades 4 y 6), se registraron algunas pipas en asociación con tiestos, restos de diversos animales, entre otros materiales (por ejemplo, uno de los bezoares), y la muy cercana presencia de un silbato confeccionado en hueso ave, muy similar a los que se utilizan en prácticas rituales chamánicas registradas entre los mataco (Dijour 1933; Califano 1976: 29, 47; Pérez Bugallo 1989: 93-95), que refuerzan dicha hipótesis. Al respecto, sobre la posible vinculación entre el uso de este aerófono y el consumo de enteógenos, Metraux (1944) señala lo siguiente sobre comunidades originarias cercanas al área de estudio: 
"[...] Los hechiceros lules y matacos son los únicos shamanes del Chaco que se ponen a sí mismos en estado de arrobamiento, por medio de tomas por la nariz de un polvo hecho de semillas de sebil (...). Cuando han alcanzado ese estado envían sus almas, en forma de pájaro, fuera del cuerpo. La metamorfosis es facilitada con los silbidos de un pito hecho de un hueso del mismisimo pájaro" (Métraux 1944: 304, citado por Pérez Gollán y Gordillo 1993: 311).

Respecto del espacio correspondiente a la porción sur de $\mathrm{EM}_{3}$, que se presenta como un lugar donde se habrían desarrollado múltiples actividades, entre las que habría que destacar aquellas relacionadas con el hábito de fumar. El registro de una estructura de piedra, de posibles fragmentos de adobe, además de artefactos de molienda, partes de vasijas y restos óseos de animales (algunos de ellos con marcas de corte), entre otros restos, podrían estar indicando la presencia de un espacio residencial donde se llevaron a cabo diversas prácticas cotidianas (como el procesamiento y consumo de alimentos). En este ámbito tal vez se llevaron a cabo prácticas fumatorias en el marco de actividades (habituales o eventuales) compartidas por el grupo doméstico, algunas de las cuales pudieron haber tenido un carácter ritual, dado el registro de micro-restos de enteógenos como el cebil en las pipas allí encontradas. Este aspecto fue enfatizado en otros estudios similares, como los efectuados en las pipas del sitio Cardonal (Valle de El Cajón, Catamarca), donde se relativizan las dicotomías entre lo doméstico y lo religioso y se enfatiza que ambos tipos de actividades pudieron haberse llevado a cabo en un mismo ámbito residencial (Bugliani et al. 2010: 234). No se descarta tampoco el uso habitual, cotidiano y personal de estos instrumentos, por ejemplo, para el consumo de tabaco en la intimidad del hogar (Scarpa y Rosso 2011). El registro de fragmentos de pipas en otros sectores del sitio, considerados también como posibles ámbitos residenciales, contribuye a reforzar estas consideraciones y a sostener que el consumo de enteógenos en pipas en Yánimas 1 no estuvo restringi- do solo a ciertos individuos (por ejemplo, chamanes) y a determinadas situaciones, sino que pudo haber sido una práctica más común y accesible a otros individuos de la población, tal vez llevada a cabo con diversos fines.

A lo largo de este trabajo, se han realizado varios aportes a la problemática del uso de enteógenos en pipas en contextos prehispánicos de las selvas meridionales del NOA. En primer lugar, dado el debate anteriormente reseñado sobre el uso de estos artefactos, es preciso destacar que, para los ejemplares analizados, tanto las huellas de uso observadas como plantas psicotrópicas identificadas en sus residuos internos demuestran el desarrollo de prácticas fumatorias de enteógenos en Yánimas 1 hacia fines del primer milenio DC. En tal sentido, se resalta también que los datos obtenidos a partir del análisis de microvestigios nos permitieron lograr una primera aproximación a la identificación de las especies consumidas por estas comunidades y las posibles actividades involucradas en los hábitos fumatorios de esta ocupación. Asimismo, la integración de estos datos con la información contextual ha permitido relacionar estos hábitos con los distintos espacios del sitio y profundizar en las prácticas desarrolladas en ellos. Por último, se destaca que debido a la escasez de estudios sobre el uso de enteógenos en contextos arqueológicos para el Piedemonte Meridional del $\mathrm{NOA}$, este trabajo constituye un punto de partida para el entendimiento y conocimiento de las prácticas fumatorias en esta área de estudio.

\section{Agradecimientos}

Este trabajo fue posible gracias a la beca otorgada por el Programa Estímulo a las Vocaciones Científicas (Consejo Interuniversitario Nacional), en el marco del Proyecto CIUNT 26/G425; y extendemos nuestro agradecimiento al Laboratorio de Arqueobotánica del Instituto de Arqueología y Museo - ISES CONICET, por el uso de las instalaciones y equipos. 


\section{* Referencias citadas}

ANDREONI, D. 2010. Propuesta metodológica para reconocer las prácticas asociadas al uso de pipas arqueológicas. Actas del XVII Congreso Nacional de Arqueología Argentina, Tomo III, pp. 12121216. Mendoza.

ANDREONI, D. F.; R. C. SPANO y V. LEMA. 2012. Nota sobre evidencias del uso de plantas en el Sitio Soria 2 a partir del análisis microscópico del contenido de pipas. Arqueología 18: 235-243.

BABOT, M. P. 2004. Tecnología y utilización de artefactos de molienda en el noroeste prehispánico. Tesis doctoral, Facultad de Ciencias Naturales e IML, Universidad Nacional de Tucumán, Tucumán.

BABOT, M. P. 2009. La cocina, el taller y el ritual. Explorando las trayectorias del procesamiento vegetal en el Noroeste Argentino. Darwiniana 47(1): 7-30.

BOMAN, E. 1932. Pipas de fumar de los indígenas de la Argentina. Anales del Museo de Historia Natural "Bernardino Rivadavia" XXXV: 309-341.

BONNIN, M. y A. LAGUENS. 2000. Esteros y algarrobales. Las sociedades de las Sierras Centrales y la llanura santiagueña. En Nueva Historia Argentina. Los Pueblos Originarios y La Conquista, M. Tarragó (Comp.), pp. 147-186. Editorial Sudamericana, Buenos Aires.

BREGANTE, O. 1926. Ensayo de clasificación del Noroeste argentino. Tesis doctoral. Facultad de Filosofía y Letras. Universidad de Buenos Aires.

BUGLIANI, M. F., M. C. CALO y M.C. SCATTOLIN. 2010. Fumando en la cocina. Determinación de contenidos por técnicas fisicoquímicas en dos pipas cerámicas del Sitio Cardonal. En La arqueometría en Argentina y Latinoamérica, S. Bertolino, R. Cattáneo y A. Izetab (Eds.), pp. 231-236. Editorial de la Facultad de Filosofía y Humanidades, Córdoba.

CALIFANO, M., 1976. El chamanismo mataco. Scripta Ethnologica 3(2): 7-60.

CANTI, M. G., 1997. An investigation of microscopic calcareous spherulites from herbivore dungs. Journal of Archaeological Science 24: 219-231.

CAPPARELli, A., M.L. POCHETTINO, D. ANDREONI y R. ITURRIZA. 2006. Differences between written and archaeological record. The case of plant micro-remains at a Northwestern Argentinean Pipe. Proceedings of the IVth International Congress of Ethnobotany (ICEB 2005), Tomo 6, pp. 397-406. Estambul (Turquía).
CLÉRICI, S., C. TORCHÁN, G. DI BÁRBARO y S. TORCHÁN. 2012. Estudio de la variabilidad genética de plantas nativas en el Distrito Chaqueño Árido de la Provincia de Catamarca. Biología enAgronomía 2 (2): 26-41.

COIL, J., KORSTANJE A., ARCHER S., y C. A. HASTORF. 2003. Laboratory goals and considerations for multiple microfossil extraction in archaeology. Journal of Archaeological Science 30: 9911008

CONSTANTINO, M. T. 1998. Psychoactive substances in the archaeology of Northern Chile and NW Argentina a comparative review of the evidence. Chungara 30 (1): 49-63.

CPN Working Group: Madella, M., A. Alexandre y T. Ball, 2005. International code for phytolith nomenclature 1.o. Annals of Botany 96 (2):253-260.

DIJOUR, E. 1933. Les cérémonies d'expulsions des maladies chez les Matako. Journal de la Sociétè des Américanistes, XXY.

DOUGHERTY, B. 1972. Las pipas de fumar de Jujuy. Relaciones de la Sociedad Argentina de Antropología VI: 83-89.

DOUGHERTY, B. 1977. Análisis de la variación cerámica en el complejo San Francisco. Obra del Centenario del Museo de la Plata, Tomo II: $237-252$

FALABELLA, F., M.T. PLANELLA y B. TAGLE. 2001. Pipes and smoking tradition in the prehispanic societies of the early ceramic period of the central region of Chile. Eleusis 5: 137-151.

FERNÁNDEZ DISTEL, A. 1980. Hallazgos de pipas en complejos precerámicos del borde de la Puna jujeña (República Argentina) y el empleo de alucinógenos por parte de las mismas culturas. Estudios Arqueológicos 5:55-75.

FERNÁNDEZ DISTEL, A. 1997. Jujuy. Diccionario arqueológico. Editorial Milor, Salta.

FERNÁNDEZ DISTEL, A., M. G. RAVIÑA y B. BALLESTA. 1999. Las pipas precolombinas del Noroeste Argentino. Academia Nacional de la Historia, Buenos Aires.

GÓMEZ AUGIER, J., G. MIGUEZ y M. CARIA. 2007. La exploraçao de sal no sector das terras baixas do Noroeste Argentino durante o Formativo: ¿Espaço de convergencia cultural?», Canindé, Revista do Museo de Arqueologia de Xingó 10: 191-216.

GONZÁLEZ, A. R. 1961-1964. La cultura de La Aguada del N. O. Argentino. Revista del Instituto de Antropología II-III: 203-253. 
GONZÁLEZ, A. R. 1963. Cultural development in Northwest Argentina. Cultural Development in Latin America. Smithsonian Misc. Coll 146, num 1.

GONZÁLEZ, A. R. 1977. Arte Precolombino de la Argentina. Introducción a su Historia Cultural. Filmediciones Valero, Buenos Aires.

GONZÁLEZ, A. R. 1998. Cultura La Aguada. Arqueología y Diseños. Filmediciones Valero, Buenos Aires.

HEREDIA, O. R. 1970. La cultura Candelaria. Rehue 3.

KRAPOVICKAS, P. 1955. El yacimiento de Tembenquiche (Puna argentina). Anales de Arqueología y Etnología, XIV-XV.

KORSTANJE, M. A. y M. P. BABOT. 2007. A microfossil characterization from South Andean economic plants. En Plants, people and places: recent studies in phytholithic analysis, M. Madela y D, Zurro (Eds.), pp. 41-72. OxbowBooks, Cambridge.

LEMA, V. S, D. ANDREONI, A. CAPPARELLI, G. ORTIZ, R. SPANO, M. QUESADAy F. ZORZI. 2015. Protocolos y avances en el estudio de residuos de pipas arqueológicas de Argentina. Aportes para el entendimiento de metodologías actuales y prácticas pasadas. Estudios Atacameños 51: 77-97.

LOY, T. 1994. Methods in the analysis of starch residues on prehistoric stone tools. En Tropical Archaeobotany: applications and new development, J.Hather (Ed.), pp. 86-114. Routledge, New York.

MANASSE, B. 1997. La región pedemontana del Sudoeste de la Provincia de Tucumán. Dptos. de Alberdi y La Cocha. Shincal 6: 141-152.

MÁRQUEZ MIRANDA, F. 1946. Los diaguitas. Revista del Museo de La Plata (Nueva Serie) 3: 186-189.

MARTEL, A., V. URQUIZA, G. MIGUEZ y J. GÓMEZ AUGIER. 2007. Informe de rescate en el sitio Yánimas 1, Dpto. La Cocha, Tucumán. Universidad de Tucumán. (Documento inédito).

METRAUX, A. 1944. Estudios de etnografía chaquense. Anales del Instituto de Etnografía Americana, Tomo V.

MIGUEZ, G. 2010. Paisaje y espacialidades del sitio Yánimas I (provincia de Tucumán). Libro de Actas del XVII Congreso Nacional de Arqueología Argentina, Tomo II, pp. 449-454. Mendoza.

MIGUEZ, G. 2014. Brillan en la selva. Contexto y análisis técnico de objetos de oro hallados en un sitio prehispánico del piedemonte meridional tucumano. Relaciones de la Sociedad Argentina de Antropología 39(1): 277-284.
MIGUEZ, G. y M. CARIA. 2015. Paisajes y prácticas sociales en las selvas meridionales de la provincia de Tucumán $\left(1^{\circ}\right.$ milenio DC). En Crónicas materiales precolombinas. Arqueología de los primeros poblados del Noroeste Argentino, M. A. Korstanje, M. Lazzari; M. Basile, M. F. Bugliani, V. Lema, L. Pereyra Domingorena y M. Quesada (Eds.). Ediciones de la SAA, pp. 111-148.

MIGUEZ, G., P. CUENYA y M. CARIA. 2012. Observaciones arqueopedológicas vinculadas a una ocupación prehispánica registrada en el sitio Yánimas i (Tucumán)". Actas del V Congreso Argentino de Geomorfología y Geología del Cuaternario, pp. 61-70. Río Cuarto.

MIGUEZ, M., N. NASIF, M. GUDEMOS y S. BERTELLI. 2013. Aves, sonidos y chamanes. Estudio interdisciplinario de un instrumento musical óseo procedente de una ocupación prehispánica de las selvas meridionales del noroeste de Argentina. Anales del Museo de América 21: 137-168.

MUÑOZ, O. y R. PEÑA. 2009. Investigaciones recientes en la determinación de residuos de pipas arqueológicas de Chile central. Boletín del Museo Nacional de Historia Natural 58: 83-89.

NASIF, N. y G. MIGUEZ. 2014. La fauna relacionada a una comunidad prehispánica del piedemonte meridional de la Provincia de Tucumán (Argentina). Folia Histórica del Nordeste 22: 203-232.

NORDENSKIÖLD, E. 1993 [1903]. Lugares precolombinos de asentamiento y entierro en la frontera sudoeste del Chaco. Traducción del original en sueco de 1903 por Alicia Fernandez Distel y Ana Distel, Serie Jujuy en el pasado, Universidad Nacional de Jujuy, Jujuy.

NÚÑEZ REGUEIRO, V. A. 1998. Arqueología, historia y antropología de los sitios de Alamito. Ediciones Interdea, San Miguel de Tucumán.

PALAMARCZUK, V., R. SPANO, F. WEBER, D. MAGNÍFICO, S. LÓPEZ y M. MANASIEWICZ. 2007. Soria 2. Apuntes sobre un sitio Formativo en el valle de Yocavil (Catamarca, Argentina). Intersecciones en Antropología 8:121-134.

PANTORRILLA, M. y V. NUÑEZ REGUEIRO. 2006. Investigaciones arqueológicas en la zona de Escaba, provincia de Tucumán: asentamientos Condorhuasi y Aguada en las Yunga. Intersecciones en Antropología 7: 235-245.

PEARSALL, D. M.y E. H. DINAN. 1992. Developing and phytolith classification system. Phyitolith Sistematics, Emerging Issues. Edited by Rapp y Mulholland. University of Minnesota, Duluth, Minnesota.

PÉREZ BUGALLO, R. 1989. Los silbatos chaquenses. Relaciones de la Sociedad Argentina de Antropología, 17 (2): 87-97. 
PÉREZ GOLLÁN, J.A y I. GORDILLO. 1993. Alucinógenos y sociedades indígenas del noroeste argentino. Anales de Antropología 30(1): 299-350.

PÉREZ GOLLÁN, J. A y I. GORDILLO. 1994. Vilca/Uturuncu. Hacia una arqueología del uso de alucinógenos en las sociedades prehispánicas de los Andes del Sur. Cuicuilco 1: 99-140.

PLANELLA, M.T., F. FALABELLA y B. TAGLE. 2000. Complejo fumatorio del período Alfarero Temprano en Chile central. Contribución Arqueológica 5: 895-909.

PLANELLA, M.T., C. BELMAR, L. QUIROZ y D. ESTÉVEZ. 2012. Propuesta integradora para un estudio del uso de plantas con propiedades psicoactivas en pipas del Período Alfarero Temprano y sus implicancias sociales. Revista de Antropología 25 93-119.

POCHETTINO, M. L. yA. CAPPARELLI. 2004. Arqueobotánica y plantas medicinales. Proceedings of the 3 rd International Symposium of Ethnobotany Disciplines, Porto Alegre (Brasil).

POCHETTINO, M., A. CORTELLA y M. RUIZ. 1999. Hallucinogenic snuff from Northwestern Argentina: microscopicalidentification of Anadenanthera columbrina var. cebil (Fabaceae) in powdered archaeological material. Economic Botany 53(2):127132.

QUIROZ, L D. 2014. Colección de referencia de microrrestos de las especies nativas del género Nicotiana en Chile. Resúmenes del Taller "Micro paleoetnobotánica: Relevancia de una red interdisciplinaria de investigaciones en fitolitos y almidones". La Paloma, Uruguay, 8 al 12 de Diciembre de 2014.

QUIROZ, L. D., C. A. BELMAR, M. T. PLANELLA, R. MERA y D MUNITA. 2012. Estudio de microfósiles de residuos adheridos en pipas cerámicas del Sitio Villa JMC-1 Labranza, región de la Araucanía1. Magallania 40:249-261.
RENDACE, S., D. ARGAÑARÁZ FOCHI, A. CORDOMÍ y P. CUENYA. 2006. Pedología y niveles de ocupación arqueológica. Actas $3^{\circ}$ Congreso Argentino de Cuaternario y Geomorfología, Actas 3, pp. 827-836. Córdoba.

ROSSO, C. y R. SPANO. 2005-2006. Humos del vecino. Evidencias del uso de alucinógenos en pipas halladas en dos sitios tempranos de los Valles Calchaquíes. Arque ología 13: 79-98.

RYDEN, S. 1936. Archaeological Researches in the Department of La Candelaria (provincia Salta, Argentina). Ethnological Studies, 3.

SCARPA, G. y C. ROSSO. 2011. Etnobotánica del "coro" (Nicotiana paa, Solanaceae): Un tabaco silvestre poco conocido del extremo sur de Sudamérica. Bonplandia 20(2): 391-404.

SCHULTES, R. y A. HOFMANN. 2000 [1982]. Plantas de los Dioses. Orígenes del uso de los alucinógenos. Fondo de Cultura Económica, México.

SERRANO, A. 1934. El uso de vegetales narcotizantes entre los indígenas de América. Revista Geográfica Americana 2: 415-428.

SINGH, N. 2003. Mito-Alucinógenos: relación y evidencia en la producción artística precolombina. Trabajo de Tesis para optar al título de Magister en Historia del Arte, Facultad de Filosofía y Letras, Universidad Nacional de Tucumán, Tucumán.

TARTUSI, M., Y V. NÚÑEZ REGUEIRO. 2003. Procesos de interacción entre poblaciones de los valles intermontanos del noroeste argentino y las del piedemonte. EnAnales Nueva Época 6, P. Cornell y P. Stenborg (Eds.), pp. 43-62. Goteborg.

VELANDIA JAGUA, C. 2014. Variaciones irreverentes sobre el arte de fumar. Comechingonia Virtual 8(2): 68-87.

WILBERT, J. 1987. Tabacco and Shamanism in South America. Yale University Press, Chelsea. 